\title{
Extraction of the Third-Order $3 \times 3$ MIMO Volterra Kernel Outputs Using Multitone Signals
}

\author{
Zain Ahmed Khan ${ }^{\circledR}$, Student Member, IEEE, Efrain Zenteno ${ }^{\circledR}$, Member, IEEE, \\ Peter Händel ${ }^{\circledR}$, Senior Member, IEEE, and Magnus Isaksson, Senior Member, IEEE
}

\begin{abstract}
This paper uses multitone signals to simplify the analysis of $3 \times 3$ multiple-input multiple-output (MIMO) Volterra systems by isolating the third-order kernel outputs from each other. Multitone signals fed to an MIMO Volterra system yield a spectrum that is a permutation of the sums of the input signal tones. This a priori knowledge is used to design multitone signals such that the third-order kernel outputs are isolated in the frequency domain. The signals are designed by deriving the conditions for the offset and spacing of the input frequency grids. The proposed technique is then validated for the six possible configurations of a $3 \times 3$ RF MIMO transmitter impaired by crosstalk effects. The proposed multitone signal design is used to extract the third-order kernel outputs, and their relative contributions are analyzed to determine the dominant crosstalk effects for each configuration.
\end{abstract}

Index Terms-Crosstalk, hardware impairments, multipleinput multiple-output (MIMO), multitone signals, Volterra kernels.

\section{INTRODUCTION}

$\mathbf{T}$ HE Volterra theory has been developed over the years for the study of single-input single-output (SISO) dynamic nonlinear systems with fading memory [1]-[4]. Therefore, it is being utilized in a wide range of applications such as mechanics, communications, and biology, among others. Thus, extraction and identification of the Volterra kernels have been a topic of particular interest for the research community.

Recent advances in system theory have yielded an increase in the interest for multiple-input multiple-output (MIMO) dynamic nonlinear systems. Therefore, the MIMO Volterra series has been developed over the years [5]-[7]. Compared to SISO Volterra kernels, two types of kernels have been indicated for MIMO systems in [5], namely, the self-kernels, which are the same as the SISO Volterra kernels, and the additional crosskernels, which arise from nonlinear interactions of different inputs of the MIMO system. Furthermore, the symmetry properties of the self-kernels are same as

Manuscript received February 19, 2018; revised May 18, 2018; accepted June 30, 2018. (Corresponding author: Zain Ahmed Khan.)

Z. A. Khan and P. Händel are with the Department of Information Science and Engineering, KTH Royal Institute of Technology, 11428 Stockholm, Sweden (e-mail: zakh@kth.se).

E. Zenteno is with the Department of Electronics and Telecommunications, Universidad Catolica San Pablo, Arequipa 04001, Peru.

M. Isaksson is with the Department of Electronics, Mathematics, and Natural Sciences, Gävle University College, 80176 Gävle, Sweden.

Color versions of one or more of the figures in this paper are available online at http://ieeexplore.ieee.org.

Digital Object Identifier 10.1109/TMTT.2018.2854186 the SISO Volterra kernels. but the crosskernels cannot be symmetrized in the same way [5]. Due to these intrinsic differences, identification of MIMO Volterra kernels is more challenging than SISO Volterra kernels.

Hence, this paper presents a method that uses multitone signals to extract the third-order MIMO Volterra kernel outputs validated for a $3 \times 3 \mathrm{RF}$ MIMO transmitter. The contribution is valuable for both academia and practitioners with respect to simplifying the analysis of MIMO Volterra systems. The extracted kernel outputs can be used by system identification researchers working with dynamic nonlinear MIMO systems, by computational modelers investigating low-complexity solutions within the Volterra framework, and by RF engineers for an accurate analysis of predominant crosstalk effects prevailing in RF MIMO transmitters.

Characterization techniques for the analysis of SISO Volterra kernels have been proposed in both time [3], [8] and frequency domains [9]-[15]. Volterra kernels can be analyzed with much more simplicity of interpretation in the frequency domain than time domain [9], [10]. Therefore, two-tone and three-tone tests have been proposed, where the frequency of tones is swept for the measurement and analysis of SISO nonlinear systems [16]-[21]. A combination of two-tone and three-tone tests for the identification of MIMO Volterra kernels have been proposed in [22]. However, two-tone tests do not excite the complete Volterra kernels, whereas three-tone tests require a large number of measurements.

Therefore, signals that contain the same characteristics but with a larger number of harmonics, referred to as multitone signals, have been proposed [9]. Excitation of a Volterra system with multitone signals yields a frequency grid at the output which consists of permutations of sums of the input signal tones. This a priori information is used to measure the SISO Volterra kernels in [9]-[11], and [12]. Furthermore, a multitone signal design for a SISO nonlinear model testing has also been presented in [14].

In contrast to SISO, it can be noted that the multiple signals of an MIMO system provide an extra degree of freedom to design input signals that fulfill the desired requirements regarding intermodulation and crossmodulation products, amplitude-to-amplitude or amplitude-to-phase distortions, spectral leakage, and so on [23]. Furthermore, any $J \times K$ MIMO Volterra system can be analyzed as a decomposition of $K$ MISO systems, each with $J$ inputs [5]. Therefore, the number of inputs determines the creation of distinct kernels, 


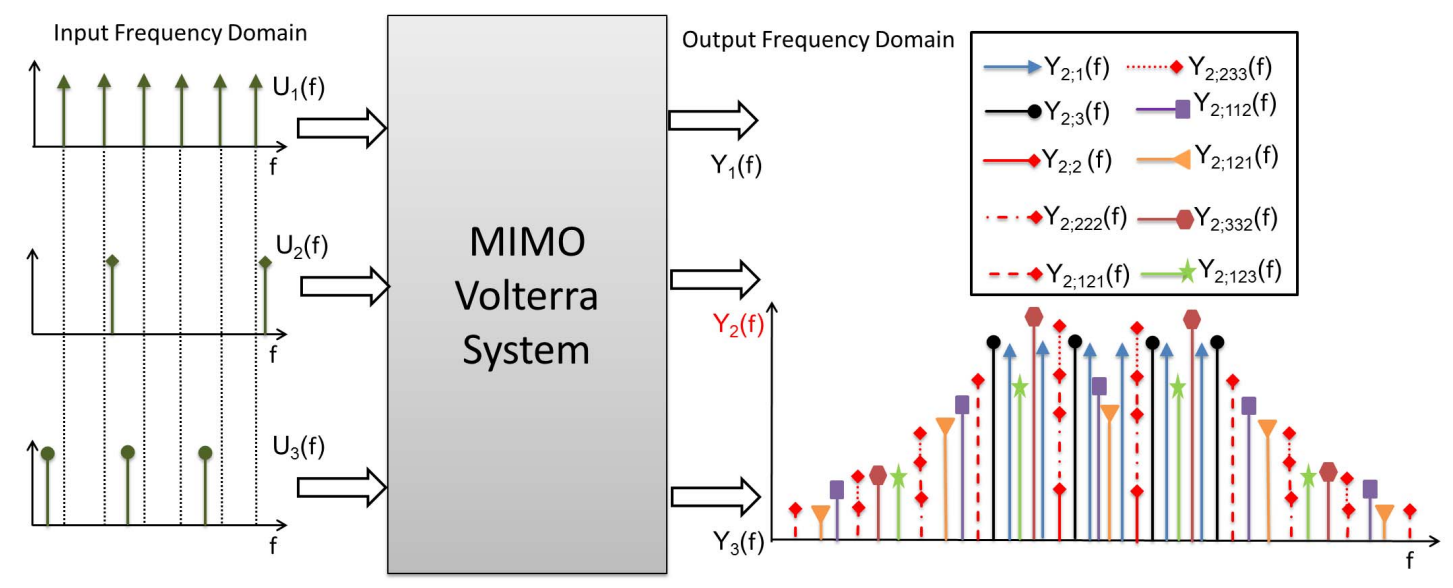

Fig. 1. Frequency isolation of the third-order kernel outputs for a $3 \times 3$ MIMO Volterra system excited by multitone signals. Only the output at channel 2 is shown as an example.

whereas the number of outputs indicates the number of times these kernels need to be considered (a set of kernels is defined for each output). These features are used to design multitone signals with differing tone spacings to isolate third-order kernel outputs for a $2 \times 2$ MIMO system in [24].

This paper extends the method presented in [24] for the extraction of the third-order $3 \times 3$ MIMO Volterra kernel outputs as shown in Fig. 1. The signal design procedure for the frequency spacing and offset is also described, extendable for any $J \times K$ MIMO system. Furthermore, the proposed method allows the use of multitone signals that resemble real operating conditions. Since the multitone design is dependent only on its tone position, the amplitudes and phases can, for instance, be made user selectable, to match the desired pdf characteristics [14].

The proposed method is validated for a $3 \times 3 \mathrm{RF}$ MIMO transmitter impaired by additional crosstalk effects that are not observable in RF SISO transmitters. Crosstalk arises from the RF leakage between different transmission paths in an MIMO transmitter being implemented on the same chipset [25]-[29]. RF leakage that occurs prior to the amplification stage is called input crosstalk, whereas RF leakage that occurs after the amplification stage is called output crosstalk [30]-[33].

A $3 \times 3$ MIMO transmitter is considered in this paper to analyze the crosstalk arising from multiple channels which are not present in a $2 \times 2$ MIMO transmitter. These additional impairments yield new scenarios for the MIMO transmitter that have not yet been analyzed, to the authors best knowledge. Thus, for a $3 \times 3 \mathrm{RF}$ MIMO transmitter with crosstalk reciprocity, there are a total of six possible configurations based on the permutations of the presence (or absence) of input and output crosstalk, as shown in Fig. 2. Furthermore, crosstalk in an MIMO transmitter decays with the distance between the channels [34], yielding strong contributions from only the adjacent channels (as noted in the experimental validation of this paper). Therefore, MIMO systems with greater than three inputs $(4 \times 4$ or higher order MIMO systems) can be studied with three inputs as subblocks of the reconsidered $3 \times 3$ MIMO systems.
This paper uses the proposed method to determine the relative contributions of the kernel outputs from their respective power spectral densities (PSDs) for each of the six configurations of a $3 \times 3$ RF MIMO transmitter (cf., Fig. 2). Crosstalk arising from the adjacent channels yields strong contributions, whereas nonadjacent channel crosstalk yields weak contributions close to the noise floor. Therefore, the relative contributions can then be used to identify the corresponding crosstalk configurations prevailing in the transmitter.

The paper is organized as follows. Section II describes the theoretical framework used in this paper. Section III presents the multitone signal design for extracting the corresponding third-order kernel outputs. An experimental investigation is performed for each of the six possible crosstalk configurations in Section IV. Finally, Sections V and VI describe discussions and conclusions, respectively.

\section{Theoretical Framework}

\section{A. MIMO Volterra Systems}

This paper studies an MIMO Volterra system with three inputs and three outputs (referred to as a $3 \times 3$ MIMO system). The system is analyzed for discrete time complex-valued baseband signals. The input and output signals are denoted as $u_{k}(n)$ and $y_{k}(n)$, respectively, where $k=1,2,3$ is the signal index; and $n=1,2, \ldots, N$ denotes the sample instant. To simplify the analysis, synchronous sampling is assumed.

Since this paper studies nonlinear effects of the $3 \times 3$ MIMO Volterra system up to the third order nonlinearities, the $k$ th output can be described using the additive properties of the MIMO Volterra system as in [28]

$$
y_{k}(n)=y_{k}^{(1)}(n)+y_{k}^{(3)}(n) .
$$

Note that only odd-order outputs are considered for complex-valued baseband signals [35]. The first term in (1) is the linear output of the MIMO system expressed as a linear combination of three FIR filters whose coefficients $h_{k ; k_{1}}^{(1 ; 3)}(m)$ 
are the linear MIMO Volterra kernels. That is,

$$
\begin{aligned}
y_{k}^{(1)}(n) & =\sum_{k_{1}=1}^{3} y_{k ; k_{1}}(n) \\
y_{k ; k_{1}}(n) & =\sum_{m=0}^{\infty} h_{k ; k_{1}}^{(1 ; 3)}(m) u_{k_{1}}(n-m)
\end{aligned}
$$

where $y_{k ; k_{1}}(n)$ is the $k$ th MIMO Volterra linear output produced by the $k_{1}$ th input. The second term in (1) is the third-order output of the MIMO system expressed as

$$
y_{k}^{(3)}(n)=\sum_{k_{1}=1}^{3} \sum_{k_{2}=k_{1}}^{3} \sum_{k_{3}=1}^{3} y_{k ; k_{1} k_{2} k_{3}}(n)
$$

where $y_{k ; k_{1} k_{2} k_{3}}(n)$ is the $k$ th MIMO Volterra third-order output produced by the $k_{1}, k_{2}, k_{3}$ input. For complex-valued baseband MIMO Volterra kernels, only the unique kernel outputs are represented in (3) as the second summation starts with $k_{2}=k_{1}$ due to symmetry of Volterra kernels [28]. Such contributions can be expressed as in [28]

$$
\begin{aligned}
y_{k ; k_{1} k_{2} k_{3}}(n)= & \sum_{m_{1}=0}^{\infty} \sum_{m_{2}=0}^{\infty} \sum_{m_{3}=0}^{\infty} h_{k ; k_{1} k_{2} k_{3}}^{(3 ; 3)}\left(m_{1}, m_{2}, m_{3}\right) \\
& \times u_{k_{1}}\left(n-m_{1}\right) u_{k_{2}}\left(n-m_{2}\right) u_{k_{3}}^{*}\left(n-m_{3}\right)
\end{aligned}
$$

where $h_{k ; k_{1} k_{2} k_{3}}^{(3 ; 3)}\left(m_{1}, m_{2}, m_{3}\right)$ are the third-order $3 \times 3$ MIMO Volterra kernels. These kernels are called self-kernels when $k_{1}=k_{2}=k_{3}$ or, otherwise, crosskernels. The indexes $k_{1}, k_{2}, k_{3}$ of the third-order $3 \times 3$ MIMO Volterra self-kernels and crosskernels listed in Table I.

Note that the self-kernels have the same symmetry properties as SISO Volterra systems while the crosskernels have different properties [5]. The crosskernels where $k_{1}=k_{2}$ can be symmetrized, however, the crosskernels where $k_{1} \neq k_{2}$ cannot be symmetrized. Thus, whenever possible, this paper expresses the self-kernels and crosskernels in their symmetric versions. Finally, to simplify the notation, when there is no chance for confusion, $u_{k}(n), y_{k}(n), y_{k ; k_{1}}(n)$, and $y_{k ; k_{1} k_{2} k_{3}}(n)$ will be denoted as $u_{k}, y_{k}, y_{k ; k_{1}}$, and $y_{k ; k_{1} k_{2} k_{3}}$, respectively.

\section{B. Hardware Impairments in RF MIMO Transmitters}

The proposed multitone extraction method is validated experimentally for a $3 \times 3 \mathrm{RF}$ MIMO transmitter. The paper focuses on hardware impairments arising from crosstalk between the channels as described in Section I.

Therefore, $\alpha$ and $\beta$ denote the input and output crosstalk arising from adjacent channels, respectively, whereas $\gamma$ and $\rho$ denote the input and output crosstalk arising from nonadjacent channels, as indicated in Fig. 2. Note that since the network follows the principle of reciprocity, the input (or output) crosstalk arising due to channel $k_{1}$ over channel $k_{2}$ is the same as the crosstalk arising due to channel $k_{2}$ over channel $k_{1}$ [34]. Also, if the channels are implemented in parallel configuration with equal distances between them, then $\gamma \approx \alpha^{2}$ and $\rho \approx \beta^{2}$ [34].

It must also be noted that input and output crosstalk may arise independently of one another. Input crosstalk may

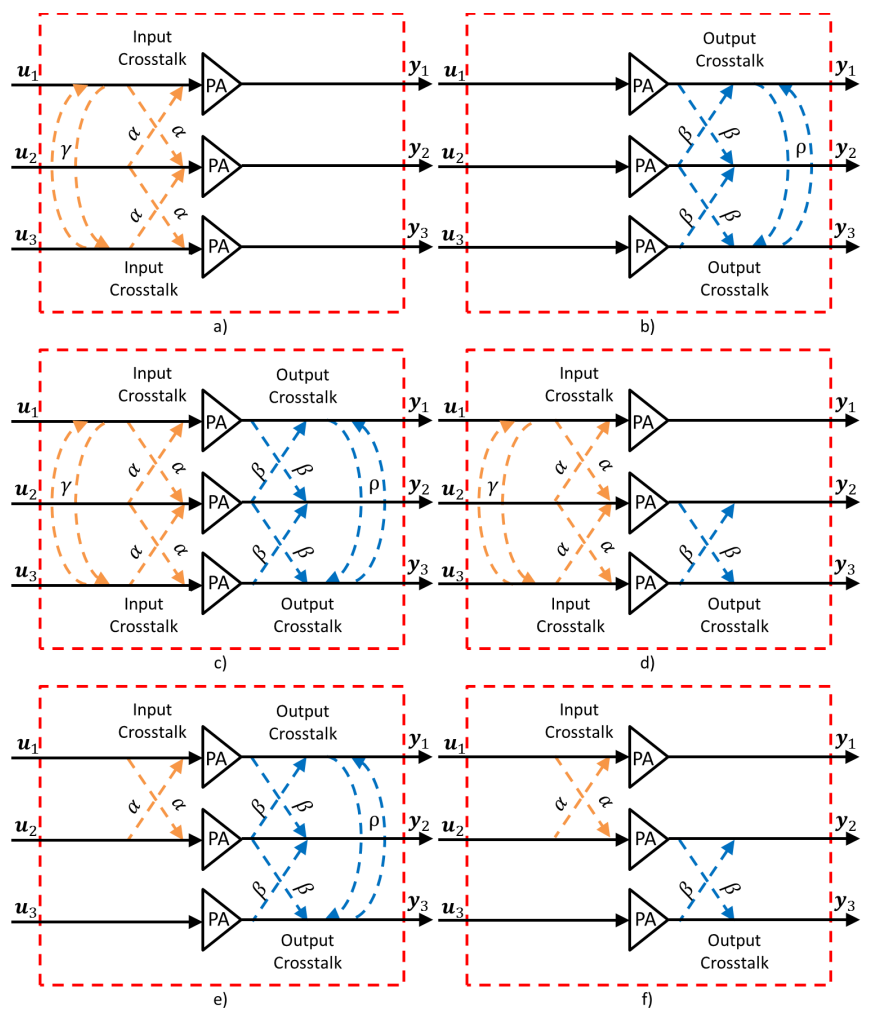

Fig. 2. Crosstalk configurations for a $3 \times 3$ RF MIMO transmitter. (a) Full-input crosstalk. (b) Full-output crosstalk. (c) Full-input full-output crosstalk. (d) Full-input partial-output crosstalk. (e) Partial-input full-output crosstalk. (f) Partial-input partial-output crosstalk.

TABLE I

TRIPlets $k_{1}, k_{2}, k_{3}$ CORRESPONDING TO THE THIRD-ORdER $3 \times 3$ MiMO Volterra Kernels $\left(h_{k ; k_{1} k_{2} k_{3}}^{(3 ; 3)}(\cdots)\right)$

\begin{tabular}{c|c}
\hline \hline Self-Kernels & Cross-Kernels \\
\hline$\{1,1,1\}$ & $\{1,1,2\},\{1,1,3\},\{1,2,1\}$ \\
$\{2,2,2\}$ & $\{1,2,2\},\{1,2,3\},\{1,3,1\}$ \\
$\{3,3,3\}$ & $\{1,3,2\},\{1,3,3\},\{2,2,1\}$ \\
- & $\{2,2,3\},\{2,3,1\},\{2,3,2\}$ \\
- & $\{2,3,3\},\{3,3,1\},\{3,3,2\}$ \\
\hline
\end{tabular}

arise from different signal sources utilizing a common local oscillator (LO). Output crosstalk may arise from antenna coupling that causes dynamic loading of the amplifiers or by using the same LO for downconversion in the feedback path. Furthermore, manufacturing imperfections such as differences in isolation between the transmission paths, differences in the fabrication of the microstrip lines and nonideal matching networks may generate crosstalk independently, either at the input or output of the amplification stage. Thus, the reciprocity and independence of input and output crosstalk yield six possible configurations for a $3 \times 3 \mathrm{RF}$ MIMO transmitter, as shown in Fig. 2.

In addition, for the analysis presented in this paper, the crosstalk is approximated as a static filter with $-20 \mathrm{~dB}$ of isolation between the adjacent channels and $-40 \mathrm{~dB}$ of isolation between the nonadjacent channels [30]. That is, 
$\alpha, \beta \approx-20 \mathrm{~dB}$, and $\gamma, \rho \approx-40 \mathrm{~dB}$. Therefore, $\alpha+\gamma \approx \alpha$, and $\beta+\rho \approx \beta$.

Furthermore, the level of input crosstalk $r_{k ; k_{1} k_{2} k_{3}}(\alpha, \gamma)$, associated with the corresponding third-order kernel outputs is

$$
r_{k ; k_{1} k_{2} k_{3}}(\alpha, \gamma)=\left[\alpha^{\sum_{\ell=1}^{3} I_{1}\left(k, k_{\ell}\right)} \gamma^{\sum_{\ell=1}^{3} I_{2}\left(k, k_{\ell}\right)}\right]
$$

where, $I_{1}\left(k, k_{\ell}\right)$ and $I_{2}\left(k, k_{\ell}\right)$ are the indicator functions for the adjacent and nonadjacent channel crosstalk, respectively, and described as

$$
I_{x}\left(k, k_{\ell}\right)= \begin{cases}1, & \text { if }\left|k-k_{\ell}\right|=x \\ 0, & \text { otherwise. }\end{cases}
$$

For example, $r_{1 ; 111}(\alpha, \gamma)=1, r_{1 ; 112}(\alpha, \gamma)=\alpha$ and $r_{1 ; 233}(\alpha, \gamma)=\alpha \gamma^{2}$.

Similarly, the level of output crosstalk $s_{k ; k_{1} k_{2} k_{3}}(\beta, \rho)$, can be described as

$$
s_{k ; k_{1} k_{2} k_{3}}(\beta, \rho)= \begin{cases}\beta^{I_{1}\left(k_{1}, k\right)} \rho^{I_{2}\left(k_{1}, k\right)}, & \text { if } k_{1}=k_{2}=k_{3} \\ 0, & \text { otherwise. }\end{cases}
$$

For example, $s_{1 ; 222}(\beta, \rho)=\beta$ and $s_{1 ; 333}(\beta, \rho)=\rho$.

Finally, the corresponding levels for input and output crosstalk $v_{k ; k_{1} k_{2} k_{3}}(\alpha, \beta, \gamma, \rho)$ are

$$
\begin{aligned}
v_{k ; k_{1} k_{2} k_{3}}(\alpha, \beta, \gamma, \rho)= & r_{k ; k_{1} k_{2} k_{3}}(\alpha, \gamma)+\beta r_{\zeta_{k} ; k_{1} k_{2} k_{3}}(\alpha, \gamma) \\
& +\rho r_{\eta_{k} ; k_{1} k_{2} k_{3}}(\alpha, \gamma)+s_{k ; k_{1} k_{2} k_{3}}(\beta, \rho)
\end{aligned}
$$

where, $\zeta_{k}$ are the adjacent channels to channel $k$ and $\eta_{k}$ are the nonadjacent channels to channel $k$. For example, $v_{1 ; 121}(\alpha, \gamma, \beta, \rho)=\alpha+\beta \alpha+\alpha^{2} \rho \gamma \approx \alpha$.

\section{Multitone Signal Design}

The goal of this paper is to design multitone excitation input signals of the form

$$
u_{k}(n)=\sum_{i=1}^{N_{k}} e^{j\left(2 \pi f_{k}(i) n+\phi_{k}(i)\right)}
$$

such that the kernel outputs (contributions) in (4) are isolated from each other. That is, they fall in distinct frequency lines for each triplet $\left\{k_{1}, k_{2}, k_{3}\right\}$. These frequency isolated contributions can then be extracted from the measured output. In (9), $j$ denotes the imaginary number, $f_{k}(i)$ and $\phi_{k}(i)$ denote the frequency and phase of the $k$ th input signal, respectively, and $N_{k}$ is the number of tones for the $k$ th input.

Note that the frequency grids of the linear MIMO kernel output in (2) are the same as the frequency grids of the input signals, i.e., $f_{k}(i)$. This is a well-known result for linear systems. Thus, in order to extract the linear MIMO Volterra kernel outputs, it is sufficient to design excitation signals with distinct frequencies.
For the third-order MIMO Volterra kernel outputs, using (9) into (4) yields

$$
\begin{aligned}
& y_{k ; k_{1}} k_{2} k_{3}(n) \\
& =\sum_{m_{1}=0}^{\infty} \sum_{m_{2}=0}^{\infty} \sum_{m_{3}=0}^{\infty} h_{k ; k_{1} k_{2} k_{3}}^{(3 ; 3)}\left(m_{1}, m_{2}, m_{3}\right) \\
& \quad \times \sum_{i_{1}=1}^{N_{k_{1}}} \sum_{i_{2}=1}^{N_{k_{2}}} \sum_{i_{3}=1}^{N_{k_{3}}} e^{j 2 \pi\left[f_{k_{1}}\left(i_{1}\right)+f_{k_{2}}\left(i_{2}\right)-f_{k_{3}}\left(i_{3}\right)\right] n} e^{j\left[\Psi_{1}+\Psi_{2}-\Psi_{3}\right]}
\end{aligned}
$$

where $f_{k_{1} k_{2} k_{3}}(\cdot, \cdot, \cdot)$ are the frequency grids of the third-order kernel outputs, $\Psi_{\ell}=\phi_{k_{\ell}}\left(i_{\ell}\right)-2 \pi f_{k_{\ell}}\left(i_{\ell}\right) m_{\ell}, \ell=1,2,3$. By comparing (10) to (9), it can be noted that the third-order MIMO Volterra kernel output is also a multitone signal that falls in a frequency grid that is a permutation of the sums of the input signal frequency grids. That is, $f_{k_{1} k_{2} k_{3}}\left(i_{1}, i_{2}, i_{3}\right)=$ $\sum_{i_{1}} \sum_{i_{2}} \sum_{i_{3}}\left[f_{k_{1}}\left(i_{1}\right)+f_{k_{2}}\left(i_{2}\right)-f_{k_{3}}\left(i_{3}\right)\right]$. Furthermore, these multitones have amplitudes equal to $\left|h_{k ; k_{1} k_{2} k_{3}}^{(3 ; 3)}\left(m_{1}, m_{2}, m_{3}\right)\right|$ and phases equal to $\Psi_{1}+\Psi_{2}-\Psi_{3}+L h_{k ; k_{1} k_{2} k_{3}}^{(3 ; 3)}\left(m_{1}, m_{2}, m_{3}\right)$. Noticing that the kernel outputs belong to specific frequency grids, input signals can be designed to ensure nonoverlapping frequency grids at the output.

\section{A. Input Grid Design}

To isolate the kernel outputs in frequency domain, this paper proposes integer equally spaced frequency grids of the input signals as

$$
f_{k}(i)=c_{k} \pm(i-1) \Delta_{k}
$$

for $i=1,2, \ldots, N_{k}$, where $c_{k}$ is an input frequency offset and $\Delta_{k}$ is an input frequency spacing. For example, $i=1$ yields $f_{k}(i)=c_{k}$, whereas $i=2$ yields $f_{k}(i)=\left\{c_{k}-\right.$ $\left.\Delta_{k}, c_{k}+\Delta_{k}\right\}$. Therefore, the multitone kernel output frequency grid $f_{k_{1} k_{2} k_{3}}(\cdot, \cdot, \cdot)$ also consists of integer equally spaced tones given as

$$
f_{k_{1} k_{2} k_{3}}(i)=c_{k_{1}, k_{2}, k_{3}} \pm(i-1) \Delta_{k_{1} k_{2} k_{3}}
$$

for $i=1,2, \ldots, N_{k_{1}} \times N_{k_{2}} \times N_{k_{3}}$, where $c_{k_{1}, k_{2}, k_{3}}$ and $\Delta_{k_{1} k_{2} k_{3}}$ are the offset and frequency spacing of the $k_{1} k_{2} k_{3}$ multitone kernel output. These values are determined as

$$
\begin{aligned}
\Delta_{k_{1} k_{2} k_{3}} & =\text { g.c.d. }\left(\Delta_{k_{1}}, \Delta_{k_{2}}, \Delta_{k_{3}}\right) \\
c_{k_{1} k_{2} k_{3}} & =c_{k_{1}}+c_{k_{2}}-c_{k_{3}}
\end{aligned}
$$

where g.c.d. denotes the greatest common divisor. Hence, by controlling the individual offsets and frequency spacings of the input signals, the location of the third-order kernel outputs can be designed, as described in the following.

1) Even-Odd Split: Previous works for SISO Volterra systems use multitone input signals with even or odd frequency grids [11], [12]. This has been extended for $2 \times 2$ MIMO systems in [24], where one input occupies an even frequency grid while the other input occupies an odd frequency grid. This causes the kernel outputs to split into even and odd frequency grids as well. For example, Table II shows this frequency splitting for a $3 \times 3$ MIMO system, where the frequency grid of 
TABLE II

SPLITTING OF THE INPUTS AND THIRD-ORdER KERNEL OUTPUTS INTO ODD AND EVEN FREQUENCY GRIDS

\begin{tabular}{c|c}
\hline \hline Odd Grid & Even Grid \\
\hline$u_{1}, u_{3}$ & $u_{2}$ \\
$y_{k ; 111}, y_{k ; 122}, y_{k ; 133}, y_{k ; 333}$, & $y_{k ; 222}, y_{k ; 121}, y_{k ; 233}, y_{k ; 112}$ \\
$y_{k ; 131}, y_{k ; 232}, y_{k ; 221}, y_{k ; 223}$ & $y_{k ; 332}, y_{k ; 123}, y_{k ; 132}, y_{k ; 231}$ \\
$y_{k ; 113}, y_{k ; 331}$ & - \\
\hline
\end{tabular}

$u_{2}$ occupies even tones whereas the other inputs $\left(u_{1}\right.$ and $\left.u_{3}\right)$, occupy odd tones. The frequency locations (even or odd) of the output multitones can be determined simply as the sums and subtractions of even and odd numbers (cf., to (10)).

2) Spacing Design: Note that for integer frequency grids, if $G$ is the number of kernel outputs required to be frequency isolated, then the following condition needs to hold

$$
\text { g.c.d. }\left(\Delta_{1}, \Delta_{2}, \Delta_{3}\right)=2 G \text {. }
$$

Therefore, $\Delta_{k}$ are multiple integers of $2 G$ and, therefore, multiples of each other.

It can be noted from Table II that there are eight kernel outputs that are required to be isolated. However, it can also be noted from (13b) that for $y_{k ; 222}, y_{k ; 121}$, and $y_{k ; 233}$, the offset $c_{222}=c_{121}=c_{233}$. Therefore, the tones in these kernel outputs are located in frequency grids that have the same offset and whose spacings are multiples of each other. These kernel outputs will always overlap each other and can only be extracted jointly. Hence, five of the kernel outputs listed in Table II can be frequency isolated individually, i.e., $y_{k ; 112}$, $y_{k ; 332}, y_{k ; 123}, y_{k ; 132}$, and $y_{k ; 231}$, whereas the remaining three kernel outputs $y_{k ; 222}, y_{k ; 121}$, and $y_{k ; 233}$ cannot be frequency isolated from each other and can be considered as one group of kernel outputs to be extracted jointly. Thus, there are six kernel outputs (five individual and one group of three) that can be frequency isolated from each other, i.e., $G=6$.

However, the overlapping kernel outputs can still be extracted if the input spacing is unequal. For example, if $\Delta_{2}=$ $4 \Delta_{1}$ and $\Delta_{3}=2 \Delta_{1}$, then $\Delta_{121}=\Delta_{1}, \Delta_{233}=2 \Delta_{1}$, and $\Delta_{222}=4 \Delta_{1}$. Therefore, $y_{k ; 121}$ will overlap only partially with $y_{k ; 233}$ and $y_{k ; 222}$, and its contribution can be determined by extracting the nonoverlapping sections of its spectrum. Furthermore, $y_{k ; 233}$ is overlapped completely by $y_{k ; 121}$, but only partially by $y_{k ; 222}$. Thus, its contribution can be determined by extracting the tones in its spectrum that do not overlap with $y_{k ; 222}$ and subtracting the already known contribution of $y_{k ; 121}$. Finally, $y_{k ; 222}$ is overlapped completely by $y_{k ; 121}$ and $y_{k ; 233}$, and its contribution can be determined by subtracting the already known contributions of $y_{k ; 233}$ and $y_{k ; 121}$.

In conclusion, the spacing design for extracting the kernel outputs, either directly or jointly, for minimum bandwidth with $G=6$ requires $\Delta_{1}=12, \Delta_{3}=24$ and $\Delta_{2}=48$. Consequently, for equal bandwidth input signals, $N_{3}=N_{1} / 2$ and $N_{2}=N_{1} / 4$.

3) Offset Design: For completely nonoverlapping kernel outputs (or partially overlapping groups), the third-order MIMO Volterra kernel outputs yielding even frequency grids are analyzed using (13b) for all possible overlaps. The results are summarized in Table III for $c_{1}$ with $c_{2} \rightarrow$ even and $c_{2} \rightarrow$ odd. It must be noted that since $y_{k ; 121}, y_{k ; 233}$, and $y_{k ; 222}$ form a partially overlapping group, the offset relations are derived only for $y_{k ; 121}$.

From Table III, the offset relations for nonoverlapping kernel outputs are

$$
\begin{aligned}
& c_{1} \neq c_{3} \pm \frac{\mu \Delta_{1}}{2} \\
& c_{1} \neq 2 c_{2}-c_{3} \pm \mu \Delta_{1} \\
& c_{1} \neq \frac{2 c_{2}+c_{3} \pm \mu \Delta_{1}}{3} \\
& c_{1} \neq 3 c_{3}-2 c_{2} \pm \mu \Delta_{1}
\end{aligned}
$$

where $\mu=1,2,3, \ldots$, denotes integer valued periods of factors of $\Delta_{1}$. It must be noted that in Table III, conditions for nonoverlap refer to the mathematical relationships obtained for the nonoverlapping kernels using (13b), whereas the relations for nonoverlap refer to the explicit mathematical relationships obtained by solving (or simplifying) the corresponding conditions for nonoverlap.

\section{B. Extraction Process}

Based on the aforementioned signal design, the kernel outputs are extracted from the a priori information regarding the tone positions of the kernel outputs. Furthermore, since any $J \times K$ MIMO system can be analyzed as a decomposition of $K$ MISO systems, each with $J$ inputs [5], the extraction process is the same for all the output channels, and is described below.

1) The MIMO system is excited with tones located in an even frequency grid for $u_{2}$, and odd frequency grids for $u_{1}$ and $u_{3}$. The spacing and offset design for the location of the tones of these inputs signals is described in Section III-A. This design yields nonoverlapping kernel outputs $y_{k ; 112}, y_{k ; 332}, y_{k ; 123}, y_{k ; 231}$, and $y_{k ; 132}$, and partially overlapping kernel outputs $y_{k ; 121}, y_{k ; 233}$, and $y_{k ; 222}$.

2) The nonoverlapping kernel outputs $y_{k ; 112}, y_{k ; 332}, y_{k ; 123}$, $y_{k ; 231}$, and $y_{k ; 132}$ can be extracted directly from their respective PSDs using comblike filters.

3) The partially overlapping kernel outputs are extracted iteratively. First, $y_{k ; 121}$ is extracted using comblike filters from the nonoverlapping tones in its spectrum. Next, $y_{k ; 233}$ is extracted from tones in its spectrum that do not overlap with $y_{k ; 222}$. Finally, $y_{k ; 222}$ is extracted from tones in its spectrum that do not overlap with the linear output $y_{k ; 2}$ (the spectral regrowth region).

4) The inputs $u_{1}$ and $u_{2}$ are swapped yielding $y_{k ; 111}$, $y_{k ; 122}$, and $y_{k ; 133}$ as the partially overlapping kernel outputs, and $y_{k ; 221}$ and $y_{k ; 331}$ as the nonoverlapping kernel outputs. Steps 2 and 3 are then repeated with these groups.

5) The inputs $u_{1}$ and $u_{2}$ are swapped back and then $u_{3}$ and $u_{2}$ are swapped with each other. Thus, $y_{k ; 333}, y_{k ; 131}$, and $y_{k ; 232}$ form the partially overlapping group, and $y_{k ; 113}$ and $y_{k ; 223}$ form the nonoverlapping group. Steps 2 and 3 are then repeated with these groups. 
TABLE III

OfFSET DESIGN FOR NONOVERLAP OF THE THIRD-ORDER MIMO VOlterRa KERNEL OUTPUTS

\begin{tabular}{c|c|c}
\hline \hline Overlapping kernel outputs & Condition for Non-overlap & Relation for Non-overlap \\
\hline$y_{k ; 121} \rightarrow y_{k ; 112}$ & $c_{2} \neq 2 c_{1}-c_{2} \pm \mu \Delta_{1}$ & $c_{1} \neq c_{2} \pm \frac{\mu \Delta_{1}}{2}$ \\
$y_{k ; 121} \rightarrow y_{k ; 332}$ & $c_{2} \neq 2 c_{3}-c_{2} \pm \mu \Delta_{1}$ & $c_{2} \neq c_{3} \pm \frac{\mu \Delta_{1}}{2}$ \\
$y_{k ; 121} \rightarrow y_{k ; 123}$ & $c_{2} \neq c_{1}+c_{2}-c_{3} \pm \mu \Delta_{1}$ & $c_{1} \neq c_{3} \pm \mu \Delta_{1}$ \\
$y_{k ; 121} \rightarrow y_{k ; 132}$ & $c_{2} \neq c_{3}+c_{1}-c_{2} \pm \mu \Delta_{1}$ & $c_{1} \neq 2 c_{2}-c_{3} \pm \mu \Delta_{1}$ \\
$y_{k ; 121} \rightarrow y_{k ; 231}$ & $c_{2} \neq c_{3}+c_{2}-c_{1} \pm \mu \Delta_{1}$ & $c_{1} \neq c_{3} \pm \mu \Delta_{1}$ \\
\hline$y_{k ; 112} \rightarrow y_{k ; 332}$ & $2 c_{1}-c_{2} \neq 2 c_{3}-c_{2} \pm \mu \Delta_{1}$ & $c_{1} \neq c_{3} \pm \frac{\mu \Delta_{1}}{2}$ \\
$y_{k ; 112} \rightarrow y_{k ; 123}$ & $2 c_{1}-c_{2} \neq c_{1}+c_{2}-c_{3} \pm \mu \Delta_{1}$ & $c_{1} \neq 2 c_{2}-c_{3} \pm \mu \Delta_{1}$ \\
$y_{k ; 112} \rightarrow y_{k ; 132}$ & $2 c_{1}-c_{2} \neq c_{3}+c_{1}-c_{2} \pm \mu \Delta_{1}$ & $c_{1} \neq c_{3} \pm \mu \Delta_{1}$ \\
$y_{k ; 112} \rightarrow y_{k ; 231}$ & $2 c_{1}-c_{2} \neq c_{3}+c_{2}-c_{1} \pm \mu \Delta_{1}$ & $c_{1} \neq \frac{2 c_{2}+c_{3} \pm \mu \Delta_{1}}{3}$ \\
\hline$y_{k ; 332} \rightarrow y_{k ; 123}$ & $2 c_{3}-c_{2} \neq c_{1}+c_{2}-c_{3} \pm \mu \Delta_{1}$ & $c_{1} \neq 3 c_{3}-2 c_{2} \pm \mu \Delta_{1}$ \\
$y_{k ; 332} \rightarrow y_{k ; 132}$ & $2 c_{3}-c_{2} \neq c_{3}+c_{1}-c_{2} \pm \mu \Delta_{1}$ & $c_{1} \neq c_{3} \pm \mu \Delta_{1}$ \\
$y_{k ; 332} \rightarrow y_{k ; 231}$ & $2 c_{3}-c_{2} \neq c_{3}+c_{2}-c_{1} \pm \mu \Delta_{1}$ & $c_{1} \neq 2 c_{2}-c_{3} \pm \mu \Delta_{1}$ \\
\hline$y_{k ; 123} \rightarrow y_{k ; 132}$ & $c_{1}+c_{2}-c_{3} \neq c_{3}+c_{1}-c_{2} \pm \mu \Delta_{1}$ & $c_{2} \neq c_{3} \pm \mu \Delta_{1}$ \\
$y_{k ; 123} \rightarrow y_{k ; 231}$ & $c_{1}+c_{2}-c_{3} \neq c_{3}+c_{2}-c_{1} \pm \mu \Delta_{1}$ & $c_{1} \neq c_{3} \pm \frac{\mu \Delta_{1}}{2}$ \\
$y_{k ; 132} \rightarrow y_{k ; 231}$ & $c_{3}+c_{1}-c_{2} \neq c_{3}+c_{2}-c_{1} \pm \mu \Delta_{1}$ & $c_{1} \neq c_{2} \pm \mu \Delta_{1}$ \\
\hline
\end{tabular}

TABLE IV

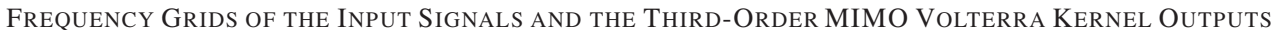

\begin{tabular}{|c|c|c|c|c|c|c|c|c|c|c|c|c|c|c|}
\hline Input signals & $\begin{array}{c}\text { Frequency Grid } \\
f_{k}(i)\end{array}$ & \multicolumn{13}{|c|}{ Example of the input signal frequency grid with $N_{k}=3$} \\
\hline$u_{1}$ & $3 \pm 12(i-1)$ & & & & -33 & -21 & -9 & 3 & 15 & 27 & 39 & & & \\
\hline$u_{2}$ & $2 \pm 48(i-1)$ & & & & -142 & -94 & -46 & 2 & 50 & 98 & 146 & & & \\
\hline$u_{3}$ & $-5 \pm 24(i-1)$ & & & & -77 & -53 & -29 & -5 & 19 & 43 & 67 & & & \\
\hline Kernel Outputs & $\begin{array}{c}\text { Frequency Grids } \\
f_{k_{1} k_{2} k_{3}(i)}\end{array}$ & \multicolumn{13}{|c|}{ Resultant excited tones of the kernel output frequency grids } \\
\hline$y_{k ; 121}$ & $2 \pm 12(i-1)$ & -214 & -202 & $\cdots$ & -34 & -22 & -10 & 2 & 14 & 26 & 38 & $\cdots$ & 206 & 218 \\
\hline$y_{k: 233}$ & $2 \pm 24(i-1)$ & -286 & -262 & ... & -70 & -46 & -22 & 2 & 26 & 50 & 74 & $\ldots$ & 290 & 314 \\
\hline$y_{k ; 222}$ & $2 \pm 48(i-1)$ & -430 & -382 & $\ldots$ & -142 & -94 & -46 & 2 & 50 & 98 & 146 & $\ldots$ & 386 & 434 \\
\hline$y_{k ; 112}$ & $4 \pm 12(i-1)$ & -212 & -200 & $\cdots$ & -32 & -20 & -8 & 4 & 16 & 28 & 40 & $\cdots$ & 208 & 220 \\
\hline$y_{k ; 332}$ & $12 \pm 24(i-1)$ & -300 & -276 & $\ldots$ & -60 & -36 & -12 & 12 & 36 & 60 & 84 & $\ldots$ & 252 & 276 \\
\hline$y_{k ; 123}$ & $10 \pm 12(i-1)$ & -242 & -230 & $\cdots$ & -26 & -14 & -2 & 10 & 22 & 34 & 46 & $\cdots$ & 250 & 262 \\
\hline$y_{k ; 132}$ & $8 \pm 12(i-1)$ & -244 & -232 & $\ldots$ & -28 & -16 & -4 & 8 & 20 & 32 & 44 & $\ldots$ & 248 & 260 \\
\hline$y_{k ; 231}$ & $6 \pm 12(i-1)$ & -246 & -234 & $\cdots$ & -30 & -18 & -6 & 6 & 18 & 30 & 42 & $\ldots$ & 246 & 258 \\
\hline
\end{tabular}

\section{Grid Design Example}

For the multitone signal design described in Section III-A, using $c_{2}=2$ and $c_{3}=-5$ in (15) yields $c_{1}=3,11$. Table IV then describes the resultant third-order kernel outputs for $c_{1}=3$ as an example. It can be noted from Table IV that although $y_{k ; 112}, y_{k ; 332}, y_{k ; 123}, y_{k ; 132}$, and $y_{k ; 112}$ have a spacing of 12 or 24 , yet each of them have different offsets. Hence, these kernel outputs are completely nonoverlapping. Furthermore, $y_{k ; 121}, y_{k ; 233}$, and $y_{k ; 222}$ have the same offset but a spacing of 12,24 , and 48, respectively. Hence, these kernel outputs form a group of partially overlapping kernel outputs. A slice of these frequency grids is shown in Fig. 3 where the amplitudes are scaled to facilitate visualization.

\section{Guidelines for $K \times K$ MIMO Volterra}

A general set of guidelines for extracting the third-order $K \times K$ Volterra kernel outputs are provided in this section.

1) The input signals are split in terms of even and odd integer valued frequency grids. Therefore, $u_{1}, u_{3}, \ldots, u_{K}$ consist of odd tones, and $u_{2}, u_{4}, \ldots, u_{K-1}$ consist of even tones, if $K$ is odd. Similarly, $u_{1}, u_{3}, \ldots, u_{K-1}$ consist of odd tones and $u_{2}, u_{4}, \ldots, u_{K}$ consist of even tones, if $K$ is even.

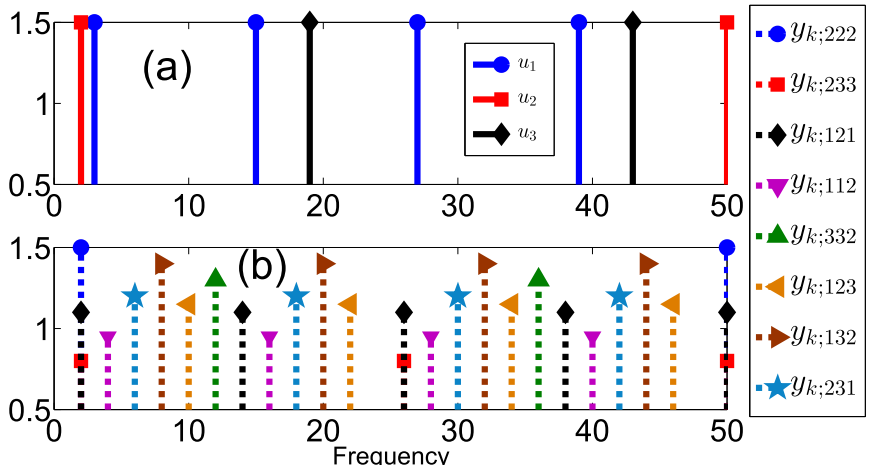

Fig. 3. Slice of the frequency grids listed in Table IV at the (a) input and (b) third-order kernel outputs. Note that the amplitudes are altered to facilitate visualization.

2) Consequently, the third-order kernel outputs are split into even and odd frequency grids as well. Each class (even and odd) is further divided into nonoverlapping kernel outputs and groups of overlapping kernel outputs, the total number of which is $G$.

3) The input spacing $\Delta_{k}$ is designed for $K$ being odd as $\Delta_{1}=2 G, \Delta_{3}=2 \Delta_{1}, \ldots, \Delta_{K}=2 \Delta_{K-2}$ and $\Delta_{2}=$ $2 \Delta_{K}, \Delta_{4}=2 \Delta_{2}, \ldots, \Delta_{K-1}=2 \Delta_{K-3}$. For $K$ being 
even, the spacing design is the same except for $\Delta_{2}=$ $2 \Delta_{K-1}$. As described in Section III-A2, $G=6$ for $K=3$. However, for $K=4, G=14$ and, therefore, $\Delta_{1}=28, \Delta_{3}=56, \Delta_{2}=112$, and $\Delta_{4}=224$. Therefore, the bandwidth efficiency of the proposed method deteriorates as $K$ increases.

4) The offset design uses an arbitrary even integer valued $c_{2}$. The conditions for the remaining offsets are then derived by analyzing all possible overlaps as described in Table III (separately for odd and even offsets). Note that for the $3 \times 3$ MIMO Volterra considered in this paper, explicit relationships are derived between $c_{1}$ and $c_{3}$. For $K=6$ as an example, the offset relations would be derived between $c_{1}, c_{3}$, and $c_{5}$, and between $c_{4}$ and $c_{6}$.

5) The kernel outputs are then extracted with comblike filters using the a priori information about the tones in the corresponding frequency grids. The corresponding extraction process is described for a $3 \times 3$ MIMO Volterra system in Section III-B. For $K=4$ as an example, $y_{141}, y_{343}, y_{242}$, and $y_{444}$ form the partially overlapping group; whereas $y_{112}, y_{114}, y_{332}, y_{334}, y_{224}, y_{442}, y_{123}$, $y_{143}, y_{132}, y_{134}, y_{231}$, and $y_{341}$ form the nonoverlapping group. The inputs $u_{2}$ and $u_{4}$ are then swapped such that $y_{121}, y_{323}, y_{244}$, and $y_{222}$ form a partially overlapping group. Finally, $u_{2}$ and $u_{3}$ are swapped, and, at the same time, $u_{4}$ and $u_{1}$ swapped, yielding nonoverlapping and partially overlapping groups for the remaining kernel outputs.

6) Note that as described in Section I, the $K \times K$ guidelines are also applicable for any $J \times K$ MIMO system [5].

\section{EXPERIMENTAL INVESTIGATION}

\section{A. Setup}

The proposed method is validated with an experimental setup that mimics a typical fourth generation (4G) $3 \times 3 \mathrm{RF}$ MIMO transmitter (cf., Fig. 4).

It must be noted that the proposed method described in Section III is designed in the digital domain, but the experiments are performed in the analog domain. Therefore, the multitone signals are created in a PC for $20-\mathrm{MHz}$ bandwidth using random phases distributed uniformly over $[-\pi, \pi]$, to generate communicationlike signals. These signals are uploaded to three Rhode and Schwartz (R\&S) SMBV100A vector signal generators (VSGs) and upconverted to $2.40 \mathrm{GHz}$ to excite the device under test (DUT).

The VSGs enhance RF phase coherency using an HS9003 signal generator from Holzworth Instrumentation [36] as a common external LO. The outputs from the DUT are downconverted to an intermediate frequency using a $2.04-\mathrm{GHz}$ LO with wideband downconverters. The downconverted signals are measured using a 2 channel 14-bit resolution analogto-digital converter (ADC) operating at a sampling frequency of $400 \mathrm{MHz}$. Note that since $J \times K$ MIMO systems can be analyzed as K MISO systems with J inputs, the measurements are performed in one output at a time, using switches for example. Therefore, an electronic switch is used in this paper
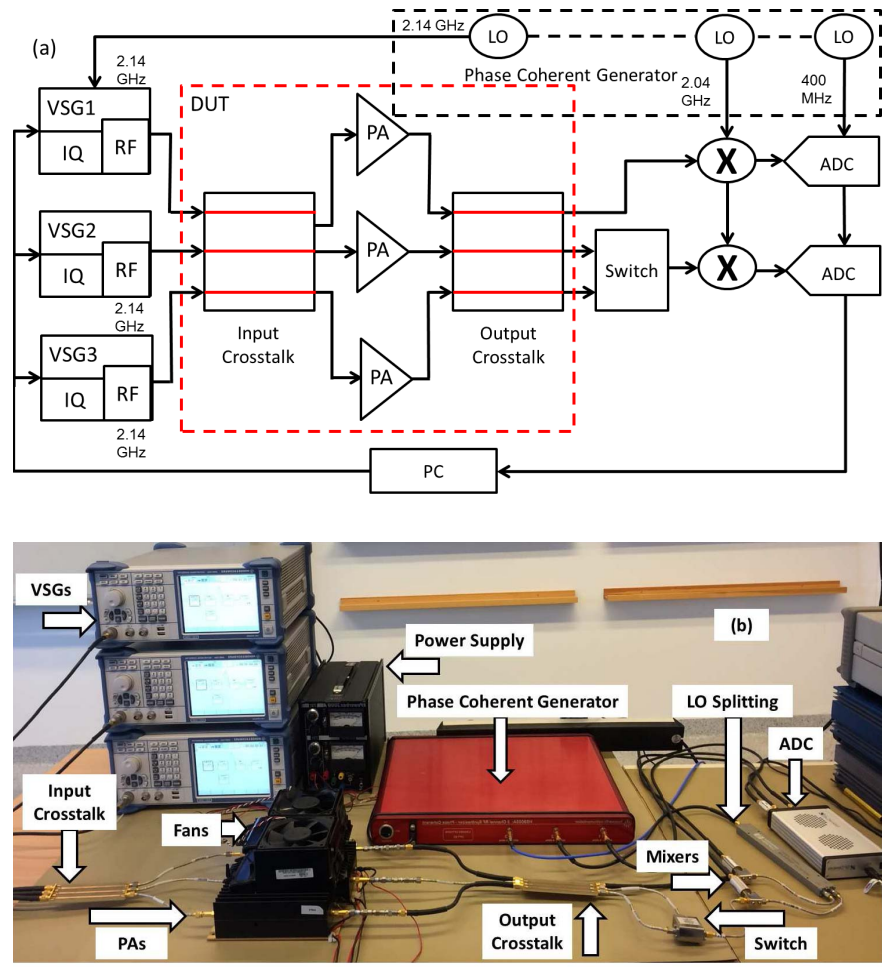

Fig. 4. (a) Outline of the measurement setup used in experiments depicting three signal sources exciting the DUT, the downconversion process, and measurement with ADC. (b) Measurement setup used in experiments.

to perform three-channel measurements with a two-channel ADC. The ADC and the VSGs are then connected through a PC for control. Finally, the measured outputs are postprocessed with subsampled synchronization in the PC to compensate for delays in time and phase [29], [37], [38].

The DUT consists of three Mini Circuits ZHL-42 PAs placed between two coupling stages used for introducing crosstalk effects [22], [27]. The PAs have a gain of $30 \mathrm{~dB}$ each and a 1-dB compression point at an output power of $30 \mathrm{dBm}$. The crosstalk stages consist of three transmission lines manufactured in parallel using an FR4 substrate, mimicking RF leakage effects, and observe -19.7 and $-37.0-\mathrm{dB}$ isolation between the adjacent and nonadjacent channels, respectively. The experiments are performed for the six crosstalk configurations described in Fig. 2.

\section{B. Results}

PSD of the extracted third-order MIMO Volterra kernel outputs for a $3 \times 3 \mathrm{RF}$ MIMO transmitter are plotted to identify the dominant hardware impairments in the different configurations. Note that since the self-kernel outputs overlap with the linear kernel outputs in-band, the contribution of these kernel outputs is depicted jointly.

1) Full Input Crosstalk: The PSD of third-order MIMO Volterra kernel outputs of channel 1 for full-input crosstalk is shown in Fig. 5. It can be noted from Fig. 5(a)-(c) that $y_{1 ; 111}$ is the dominant self-kernel output whereas the other self-kernel outputs do not contribute. 

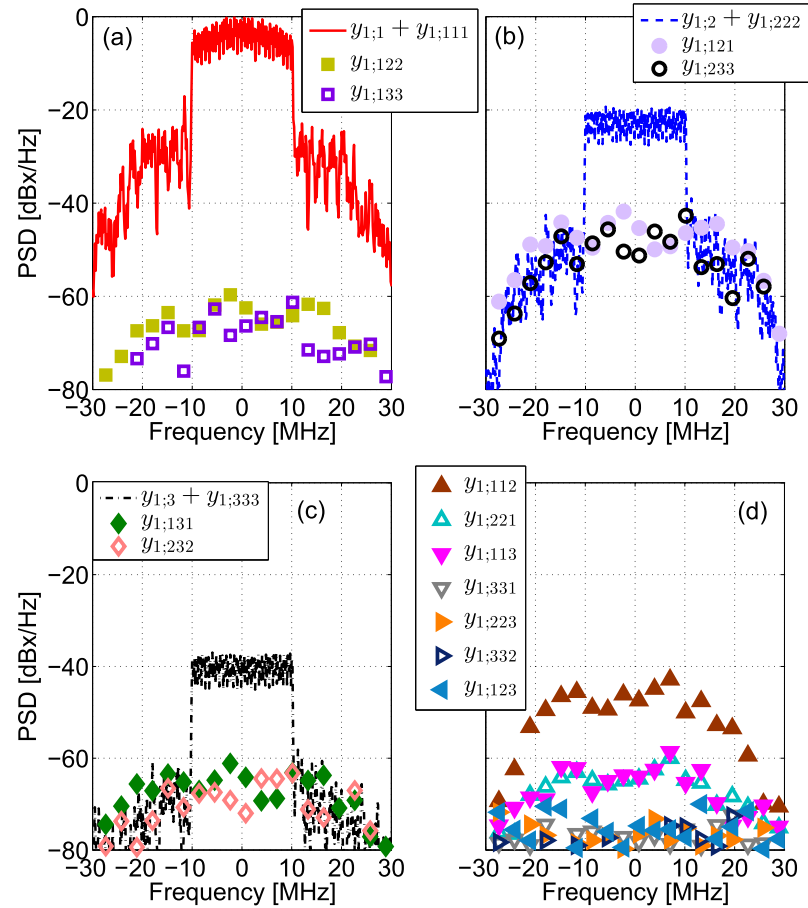

Fig. 5. PSD of the third-order kernel outputs at channel 1 for full-input crosstalk [cf., Fig. 2(a)] that partially overlap (a) $y_{1 ; 111}$, (b) $y_{1 ; 222}$, (c) $y_{1 ; 333}$, and (d) none.

For the overlapping crosskernel outputs, Fig. 5(a)-(c) shows that the crosskernel output $y_{1 ; 121}$ has a dominant contribution since it arises from adjacent channel input crosstalk. Furthermore, $y_{1 ; 122}$ and $y_{1 ; 131}$ have weak contributions close to the noise floor due to a second-order adjacent channel input crosstalk and a linear nonadjacent channel input crosstalk, respectively. The remaining overlapping crosskernel outputs do not contribute.

Finally, for the nonoverlapping crosskernel outputs, Fig. 5(d) shows that only $y_{1 ; 112}$ has a dominant contribution since it arises from adjacent channel input crosstalk. However, $y_{1 ; 221}$ and $y_{1 ; 113}$ have weak contributions close to the noise floor due to second-order adjacent channel and linear nonadjacent channel input crosstalk, respectively. The remaining nonoverlapping crosskernel outputs do not contribute.

Next, the PSD of the kernel outputs at channel 2 is shown in Fig. 6. Again, it can be noted from Fig. 6(a)-(c) that only $y_{2 ; 222}$ is the contributing self-kernel output. For the overlapping crosskernel outputs, Fig. 6(a)-(c) shows that the crosskernel outputs $y_{2 ; 122}$ and $y_{2 ; 232}$ have strong contributions due to the adjacent channel input crosstalk whereas $y_{2 ; 121}$ and $y_{2 ; 233}$ have weak contributions resulting from second-order nonadjacent channel input crosstalk. Finally, for the nonoverlapping crosskernel outputs, Fig. 6(d) shows that $y_{2 ; 221}$ and $y_{2 ; 223}$ have a strong contribution since channels 1 and 3 are both adjacent to channel 2 . However, $y_{2 ; 112}, y_{2 ; 332}$, $y_{2 ; 123}, y_{2 ; 132}$, and $y_{2 ; 231}$ have weak contributions arising from second-order adjacent channel input crosstalk. Since the kernel outputs on channel 3 can be determined by swapping $u_{1}$ with $u_{3}$ in $y_{1}$, their PSD is not plotted. Finally, it can be noted that the results presented in this section are in accordance with (5).
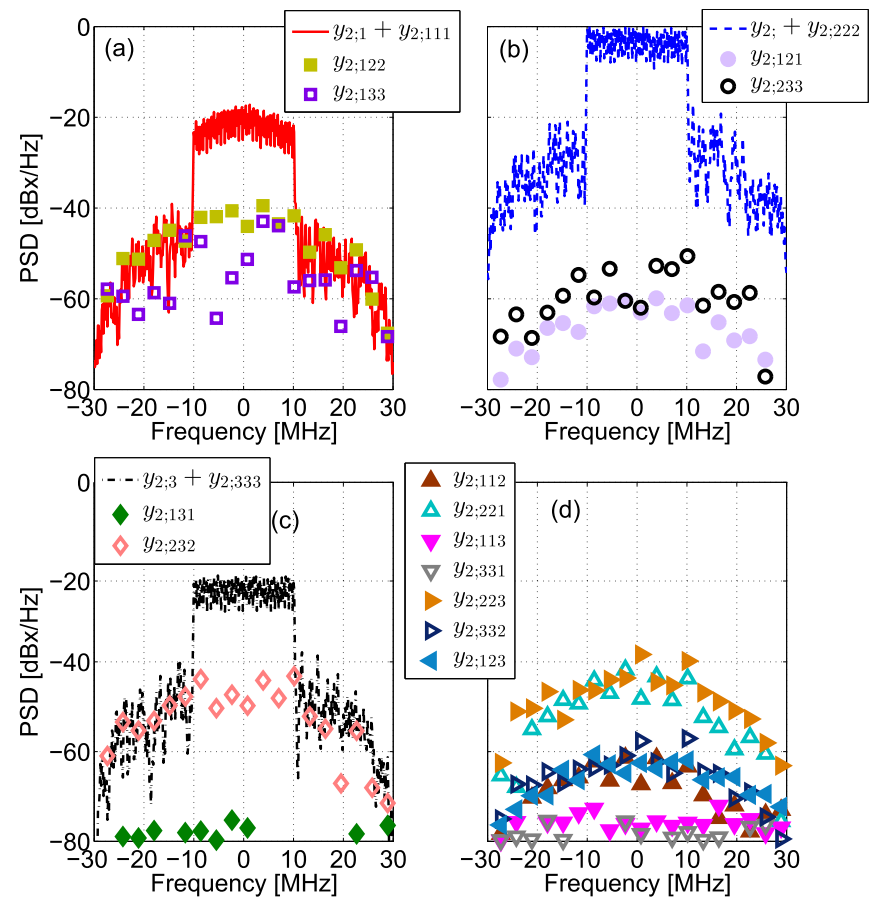

Fig. 6. PSD of the third-order kernel outputs at channel 2 for full-input crosstalk [cf., Fig. 2(a)] that partially overlap (a) $y_{2 ; 111}$, (b) $y_{2 ; 222}$, (c) $y_{2 ; 333}$, and (d) none.

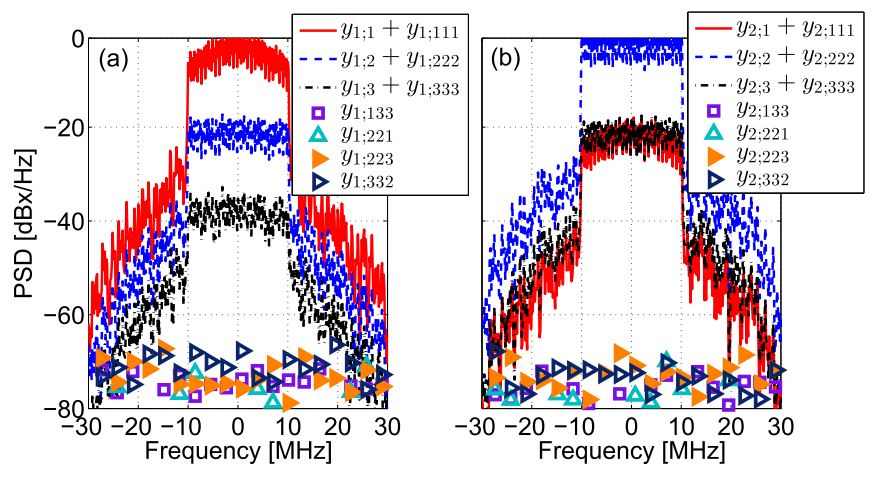

Fig. 7. PSD of third-order kernel outputs for full-output crosstalk [cf., Fig. 2(b)] at (a) Channel 1 and (b) Channel 2.

2) Full-Output Crosstalk: Fig. 7 plots the PSD of the third-order MIMO Volterra kernel outputs for a $3 \times 3$ MIMO transmitter for full-output crosstalk. It can be noted from Fig. 7 that for both channels 1 and 2, only the self-kernel outputs have a dominant contribution whereas the contribution of each of the crosskernel outputs is theoretically zero, up to the measurement noise. Furthermore, Fig. 7(a) shows that for channel $1, y_{1 ; 111}$ is the dominant self-kernel output whereas $y_{1 ; 122}$ has a strong contribution and $y_{1 ; 33}$ has a weak contribution. Finally, Fig. 7(b) shows that for channel 2, $y_{2 ; 22}$ is the strongest self-kernel output. However, the self-kernel outputs $y_{2 ; 111}$ and $y_{2 ; 33}$ have approximately equal contributions since channels 1 and 3 are both adjacent to channel 2. Again, the kernel outputs on channel 3 are not plotted due to crosstalk reciprocity with channel 1 . Finally, it can be noted that the results presented in this section are in accordance with (7). 

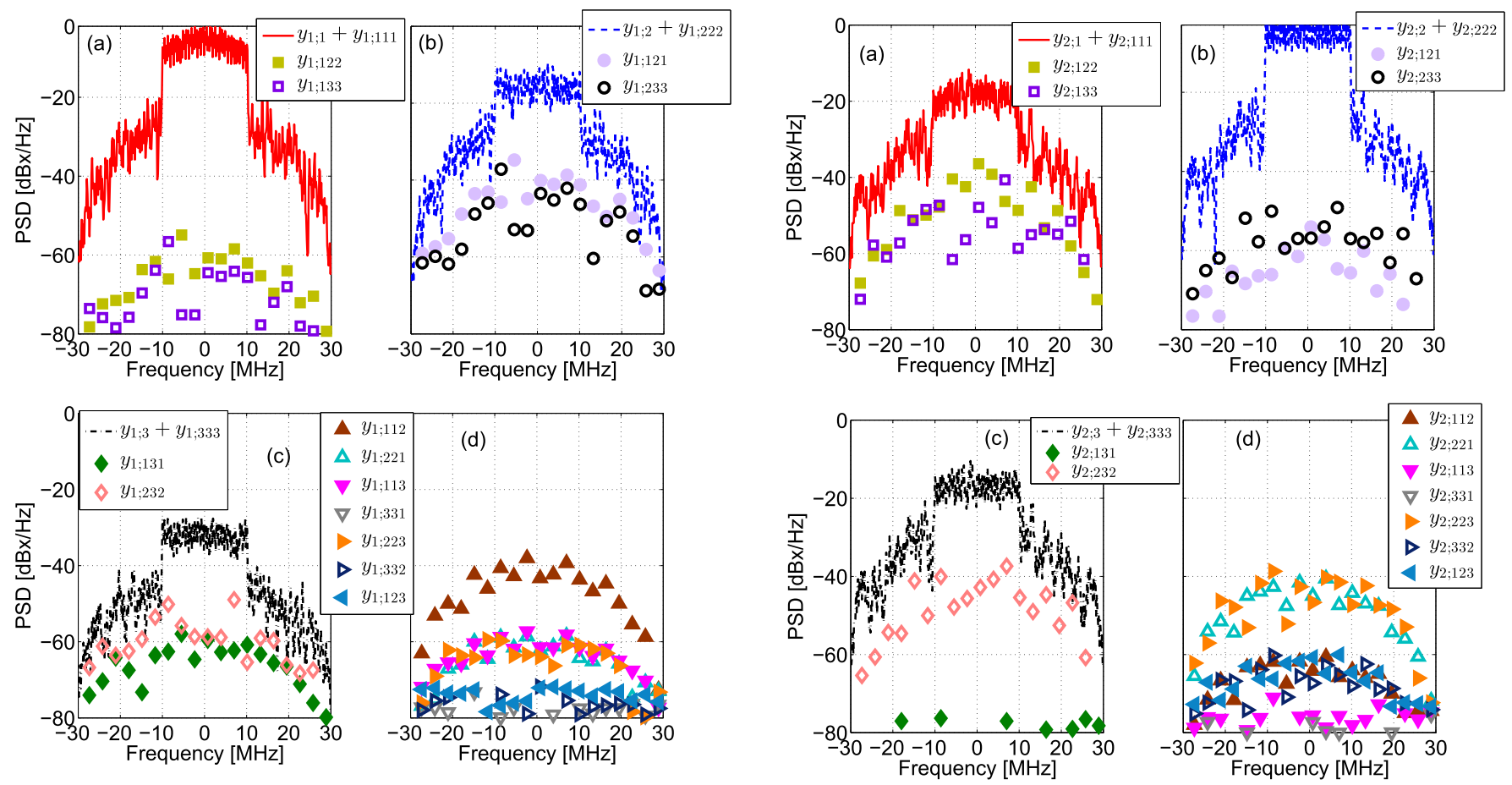

Fig. 8. PSD of the third-order kernel outputs at channel 1 for full-input fulloutput crosstalk [cf., Fig. 2(c)] that partially overlap (a) $y_{1 ; 111}$, (b) $y_{1 ; 22}$, (c) $y_{1 ; 333}$, and (d) none.

3) Full-Input Full-Output Crosstalk: Fig. 8 plots the PSD of the third-order kernel outputs at channel 1 for full-input and full-output crosstalk. It can be noted that the kernel contributions are a sum of the contributions for input and output crosstalk. However, additional crosskernel outputs $y_{1 ; 232}$ and $y_{1: 223}$ arise due to a combination of input crosstalk between channels 2 and 3 and output crosstalk between channel 1 and 2.

Next, Fig. 9 plots the PSD of the third-order kernel outputs at channel 2 for full-input and full-output crosstalk. Again, it can be noted that the kernel contributions are a sum of the contributions for input and output crosstalk. Finally, it can be noted that the results presented in this section are in accordance with (8).

\section{Identification of Crosstalk Effects}

The proposed method reveals significant differences in the output PSDs of the third-order kernel outputs, observable for each hardware configuration and in each channel (cf., Fig. 2). Therefore, the proposed method can be used to successfully identify crosstalk effects and their predominance. These contributions are summarized in Table V. The kernel outputs not listed in Table V do not contribute to the output.

\section{DISCUSSION}

From the results presented in Section VI, for the extraction of third-order $3 \times 3$ MIMO Volterra kernel outputs, the following can be noted.

1) The input grid spacing increases with the number of MIMO Volterra kernel outputs required to be extracted,

Fig. 9. PSD of the third-order kernel outputs at channel 2 for full-input fulloutput crosstalk [cf., Fig. 2(c)] that partially overlap (a) $y_{2 ; 111}$, (b) $y_{2 ; 222}$, (c) $y_{2 ; 333}$, and (d) none.

which, in turn, increase with the number of MIMO channels. Therefore, higher order MIMO systems would require wider grid spacing at the input, consequently, reducing the bandwidth efficiency of the proposed method.

2) For an RF MIMO transmitter with a large number of channels, kernel outputs up to $K-1$ nonadjacent channels contribute to the output such that the product of $\alpha^{K}$ (or $\beta^{K}$ ) with the dominant self-kernel outputs reaches the noise floor. In this paper, $\alpha \approx \beta \approx-20$ $\mathrm{dB}$, the level of the dominant self-kernel outputs is $-20 \mathrm{dBc}$, and the noise floor is at $-80 \mathrm{dBc}$. Thus, the weakest kernel outputs reach the noise floor for $K=3$. Therefore, only up to the second-order adjacent channels contribute to the output.

3) Extraction of the third-order kernel outputs does not depend on the level of crosstalk. Instead, the relative contributions of the extracted third-order kernel outputs can be used to determine the level of input or output crosstalk between two channels.

4) The proposed method can be used with any DUT that can be described as an MIMO Volterra system. Thus, the proposed method can work with different PAs and coupling hardware.

5) The proposed method can also be used in various other applications that deploy MIMO nonlinear dynamic systems, and not just RF MIMO transmitters.

6) Characterization techniques such as the proposed method provide an effective tool for obtaining system information through kernel extraction. Comparing 
TABLE V

Summary of Relative Contributions of the Third-Order MiMO Volterra Kernel OutPuts for DifFerent Crosstalk Configurations

\begin{tabular}{|c|c|c|c|c|c|c|c|c|c|}
\hline \multirow{2}{*}{ Crosstalk } & \multicolumn{3}{|c|}{ Channel 1} & \multicolumn{3}{|c|}{ Channel 2} & \multicolumn{3}{|c|}{ Channel 3} \\
\hline & Dominant & Strong & Weak & Dominant & Strong & Weak & Dominant & Strong & Weak \\
\hline \multirow{7}{*}{$\begin{array}{l}\text { Full Input } \\
\text { cf. Fig. 2(a) }\end{array}$} & $y_{1 ; 111}$ & $y_{1 ; 112}$ & $y_{1 ; 221}$ & $y_{2 ; 222}$ & $y_{2 ; 221}$ & $y_{2 ; 112}$ & $y_{3 ; 333}$ & $y_{3 ; 332}$ & $y_{3 ; 113}$ \\
\hline & - & $y_{1 ; 121}$ & $y_{1 ; 122}$ & - & $y_{2 ; 122}$ & $y_{2 ; 121}$ & - & $y_{3 ; 233}$ & $y_{3 ; 133}$ \\
\hline & - & - & $y_{1 ; 113}$ & - & $y_{2 ; 223}$ & $y_{2 ; 332}$ & - & - & $y_{3 ; 232}$ \\
\hline & - & - & $y_{1 ; 131}$ & - & $y_{2 ; 232}$ & $y_{2 ; 233}$ & - & - & $y_{3 ; 223}$ \\
\hline & - & - & - & - & - & $y_{2 ; 123}$ & - & - & - \\
\hline & - & - & - & - & - & $y_{2 ; 132}$ & - & - & - \\
\hline & - & - & - & - & - & $y_{2 ; 231}$ & - & - & - \\
\hline \multirow{2}{*}{$\begin{array}{l}\text { Full Output } \\
\text { cf. Fig. 2(b) }\end{array}$} & $y_{1 ; 111}$ & $y_{1 ; 222}$ & $y_{1 ; 333}$ & $y_{2 ; 222}$ & $y_{2 ; 111}$ & - & $y_{3 ; 333}$ & $y_{3 ; 222}$ & $y_{3 ; 111}$ \\
\hline & - & - & - & - & $y_{2 ; 333}$ & - & - & - & - \\
\hline \multirow{7}{*}{$\begin{array}{l}\text { Full Input } \\
\text { Full Output } \\
\text { cf. Fig. 2(c) }\end{array}$} & $y_{1 ; 111}$ & $y_{1 ; 112}$ & $y_{1 ; 221}$ & $y_{2 ; 222}$ & $y_{2 ; 221}$ & $y_{2 ; 112}$ & $y_{3 ; 333}$ & $y_{3 ; 332}$ & $y_{3 ; 113}$ \\
\hline & - & $y_{1 ; 121}$ & $y_{1 ; 122}$ & - & $y_{2 ; 122}$ & $y_{2 ; 121}$ & - & $y_{3 ; 233}$ & $y_{3 ; 133}$ \\
\hline & - & $y_{1 ; 222}$ & $y_{1 ; 113}$ & - & $y_{2 ; 223}$ & $y_{2 ; 332}$ & - & $y_{3 ; 222}$ & $y_{3 ; 232}$ \\
\hline & - & - & $y_{1 ; 131}$ & - & $y_{2 ; 232}$ & $y_{2 ; 233}$ & - & - & $y_{3 ; 223}$ \\
\hline & - & - & $y_{1 ; 333}$ & - & $y_{2 ; 111}$ & $y_{2 ; 123}$ & - & - & $y_{3 ; 111}$ \\
\hline & - & - & $y_{1 ; 223}$ & - & $y_{2 ; 333}$ & $y_{2 ; 132}$ & - & - & $y_{3 ; 122}$ \\
\hline & - & - & $y_{1 ; 232}$ & - & - & $y_{2 ; 231}$ & - & - & $y_{3 ; 223}$ \\
\hline \multirow{7}{*}{$\begin{array}{l}\text { Full Input } \\
\text { Partial Output } \\
\text { cf. Fig. 2(d) }\end{array}$} & $y_{1 ; 111}$ & $y_{1 ; 112}$ & $y_{1 ; 221}$ & $y_{2 ; 222}$ & $y_{2 ; 221}$ & $y_{2 ; 112}$ & $y_{3 ; 333}$ & $y_{3 ; 332}$ & $y_{3 ; 133}$ \\
\hline & - & $y_{1 ; 121}$ & $y_{1 ; 122}$ & - & $y_{2 ; 122}$ & $y_{2 ; 121}$ & - & $y_{3 ; 233}$ & $y_{3 ; 331}$ \\
\hline & - & - & $y_{1 ; 113}$ & - & $y_{2 ; 232}$ & $y_{2 ; 332}$ & - & $y_{3 ; 222}$ & $y_{3 ; 122}$ \\
\hline & - & - & $y_{1 ; 131}$ & - & $y_{2 ; 223}$ & $y_{2 ; 233}$ & - & $y_{3 ; 232}$ & $y_{3 ; 221}$ \\
\hline & - & - & - & - & $y_{2 ; 333}$ & $y_{2 ; 123}$ & - & - & $y_{3 ; 223}$ \\
\hline & - & - & - & - & - & $y_{2 ; 132}$ & - & - & - \\
\hline & - & - & - & - & - & $y_{2 ; 231}$ & - & - & - \\
\hline \multirow{4}{*}{$\begin{array}{l}\text { Partial Input } \\
\text { Full Output } \\
\text { cf. Fig. 2(e) }\end{array}$} & $y_{1 ; 111}$ & $y_{1 ; 112}$ & $y_{1 ; 221}$ & $y_{2 ; 222}$ & $y_{2 ; 221}$ & $y_{2 ; 112}$ & $y_{3 ; 333}$ & $y_{3 ; 222}$ & $y_{3 ; 122}$ \\
\hline & - & $y_{1 ; 121}$ & $y_{1 ; 122}$ & - & $y_{2 ; 122}$ & $y_{2 ; 121}$ & - & - & $y_{3 ; 221}$ \\
\hline & - & $y_{1 ; 222}$ & $y_{1 ; 333}$ & - & $y_{2 ; 111}$ & - & - & - & $y_{3 ; 111}$ \\
\hline & - & - & - & - & $y_{2 ; 333}$ & - & - & - & - \\
\hline Partial Input & $y_{1 ; 111}$ & $y_{1 ; 112}$ & $y_{1 ; 221}$ & $y_{2 ; 222}$ & $y_{2 ; 221}$ & $y_{2 ; 112}$ & $y_{3 ; 333}$ & $y_{3 ; 222}$ & $y_{3 ; 122}$ \\
\hline Partial Output & - & $y_{1 ; 121}$ & $y_{1 ; 122}$ & - & $y_{2 ; 122}$ & $y_{2 ; 121}$ & - & - & $y_{3 ; 221}$ \\
\hline cf. Fig. 2(f) & - & - & - & - & $y_{2 ; 333}$ & - & - & - & - \\
\hline
\end{tabular}

them with postprocessing techniques such as principal compressed sensing or principal component analysis (PCA), it can be noted that these methods have inherent limitations with respect to extracting system information through kernel analysis. Compressed sensing for MIMO Volterra systems is dependent on the initialization with respect to the size for the search space [39]. On the other hand, PCA is a postprocessing data technique not linked directly to a model formulation (as the Volterra series). Furthermore, it requires orthogonal search spaces, not yet developed for MIMO Volterra systems, and, thus, would require conversion from orthogonal space to Volterra kernel space [40].

\section{CONCLUSION}

This paper presents a method for the extraction of the third-order $3 \times 3$ MIMO Volterra kernels outputs using multitone excitation signals with differing tone spacing. The proposed input frequency grids yield third-order kernel outputs that fall in a nonoverlapping frequency grid, either partially or completely. It is shown that the spacing of the input signals must be an integer multiple of twice the total number of nonoverlapping kernel outputs (or partially overlapping kernel output groups). Furthermore, the relationships between the frequency offsets that ensure nonoverlapping kernel outputs are also derived. The nonoverlapping (or partially overlapping) frequency grids at the output can then be used to determine the relative strengths of the individual kernel contributions.

The proposed method is validated with measurements for the six possible crosstalk configurations of a $3 \times 3 \mathrm{RF}$ MIMO transmitter. The relative contributions of the kernel outputs are determined from their respective PSDs for each configuration. The relative kernel contributions show that input crosstalk is responsible for the generation of crosskernel outputs whereas output crosstalk is responsible for additional self-kernels arising from different adjacent or nonadjacent channels. Furthermore, it can also be noted that dominant third-order kernel outputs arise due to adjacent channel crosstalk. However, weak third-order kernel outputs arise due to a second-order adjacent channel crosstalk, a nonadjacent channel crosstalk or a combination of adjacent channel input and output crosstalk.

The extracted kernel outputs can be used for identifying the individual MIMO Volterra kernels revealing the nature of interactions between the different frequency dependent inputs 

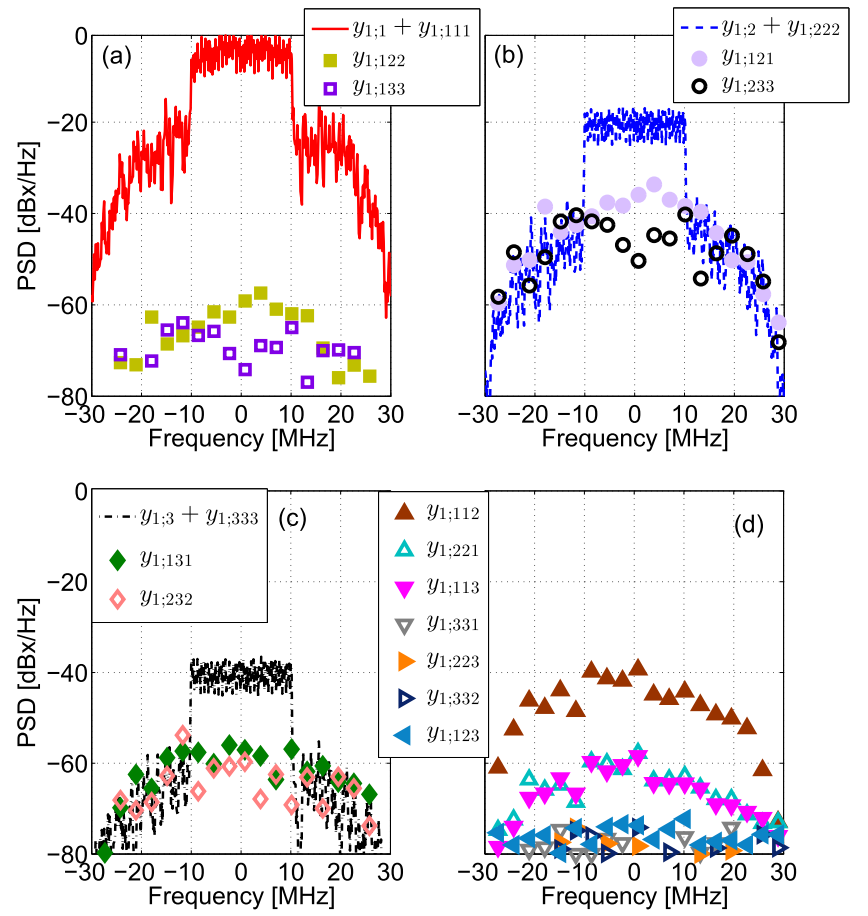

Fig. 10. PSD of the third-order kernel outputs at channel 1 for full-input partial-output crosstalk [cf., Fig. 2(d)] that partially overlap (a) $y_{1 ; 111}$, (b) $y_{1: 222}$, (c) $y_{1: 333}$, and (d) none.

of a nonlinear MIMO system. The nonoverlapping kernel can be identified directly from the respective kernel outputs using least squares estimation techniques. The partially overlapping kernels can be identified from the nonoverlapping tones of the respective kernel outputs as band-limited MIMO Volterra systems cascaded to comblike filters. Furthermore, since the proposed method reveals the dominant crosstalk effects for each configuration, the extracted kernel outputs can then be used to identify the crosstalk configuration prevailing in an MIMO transmitter. This can be particularly useful in RF massive MIMO transmitters where the identification of the crosstalk configuration between different channels becomes more significant than the actual identification of an unfeasibly large number of MIMO Volterra kernels. Finally, this paper paves the way for analyzing the kernel outputs in massive MIMO transmitters using blocks of limited inputs, i.e., using $3 \times 3$ RF MIMO subsystems as the one studied in this paper.

\section{APPENDIX}

The relative contributions of the extracted third-order MIMO Volterra kernel outputs for the crosstalk configurations depicted in Fig. 2(d)-(f) are described by plotting the PSD of each of the kernel outputs, in the following.

1) Full-Input Partial-Output Crosstalk: Fig. 10 plots the PSD of the third-order kernel outputs at channel 1 for full-input partial-output crosstalk. All the third-order kernel outputs follow the same trends as channel 1 in Fig. 5 for full-input crosstalk, since there is no output crosstalk on channel 1.

Fig. 11 plots the third-order kernel output PSD at channel 2 for full-input partial-output crosstalk. All the third-order kernel
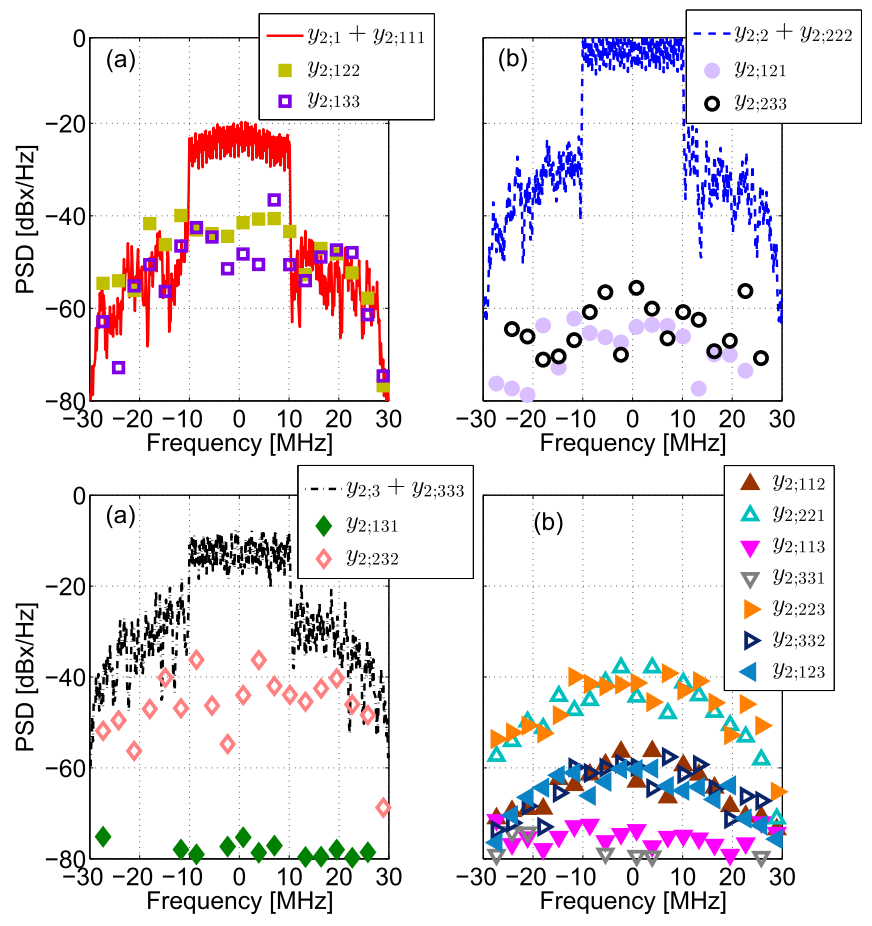

Fig. 11. PSD of the third-order kernel outputs at channel 2 for full-input partial-output crosstalk [cf., Fig. 2(d)] that partially overlap (a) $y_{1 ; 111}$, (b) $y_{1 ; 222}$, (c) $y_{1 ; 333}$, and (d) none.

outputs follow the same trends as described for channel 2 in Fig. 9 for full-input full-output crosstalk, except the kernel output $y_{2 ; 111}$, which does not contribute since there is no output crosstalk on channel 1.

Fig. 12 plots the third-order kernel PSD at the output of channel 3 for full-input partial-output crosstalk. It can be noted that the self-kernel output $y_{3 ; 222}$ has a strong contribution since it arises from adjacent channel output crosstalk, whereas the self-kernel output $y_{3 ; 111}$ does not contribute since there is no output crosstalk on channel 1. Furthermore, it can be noted that the crosskernel outputs $y_{3 ; 233}$ and $y_{3 ; 332}$ have strong contributions to the output whereas $y_{3 ; 232}, y_{3 ; 223}$, $y_{3 ; 133}, y_{3 ; 331}, y_{3 ; 121}$, and $y_{3 ; 221}$ have weak contributions to the output.

2) Partial-Input Full-Output Crosstalk: Fig. 13 plots the PSD of the third-order kernel outputs at channel 1 for partial-input full-output crosstalk. The contributions of the kernel outputs follow the same tends as full-input full-output crosstalk (cf., Fig. 8), except that the crosskernel outputs arise only form channel 2 since there is no input crosstalk on channel 3.

Fig. 14 plots the PSD of the third-order kernel outputs at channel 2 for partial-input full-output crosstalk. Again, the contributions of the kernel outputs follow the same trends as for full input full output crosstalk (cf., Fig. 9), except that the crosskernel outputs arise only from channel 1 since there is no input crosstalk on channel 3.

Finally, Fig. 15 plots the PSD of the third-order kernel outputs at channel 3 for partial-input full-output crosstalk. It can be noted from Fig. 15(a)-(c) that the self-kernel outputs follow the same trends as described for channel 1 

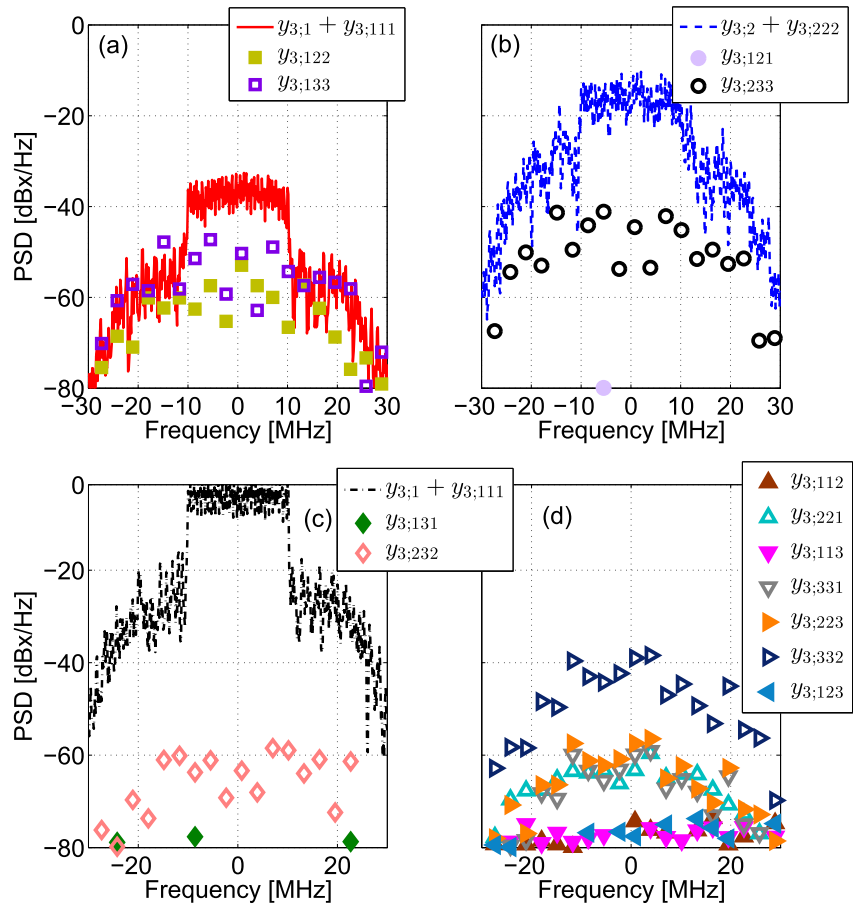

Fig. 12. PSD of the third-order kernel outputs at channel 3 for full-input partial-output crosstalk [cf., Fig. 2(d)] that partially overlap (a) $y_{2 ; 111}$, (b) $y_{2 ; 222}$, (c) $y_{2 ; 333}$, and (d) none.
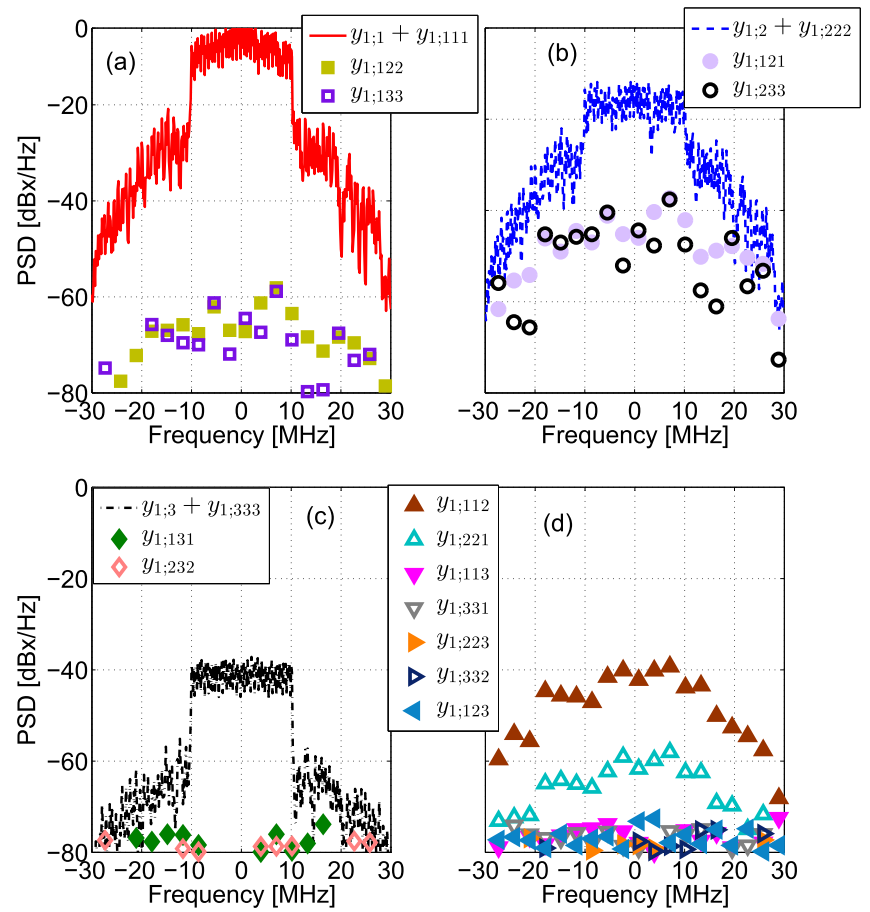

Fig. 13. PSD of the third-order kernel outputs at channel 1 for partial-input full-output crosstalk [cf., Fig. 2(e)] that partially overlap (a) $y_{1 ; 111}$, (b) $y_{1 ; 222}$, (c) $y_{1: 333}$, and (d) none.

in Fig. 13(a)-(c) due to output crosstalk reciprocity between channels 1 and 3. However, since there is no input crosstalk on channel 3 , only $y_{3 ; 221}$ and $y_{3 ; 122}$ contribute to the output.
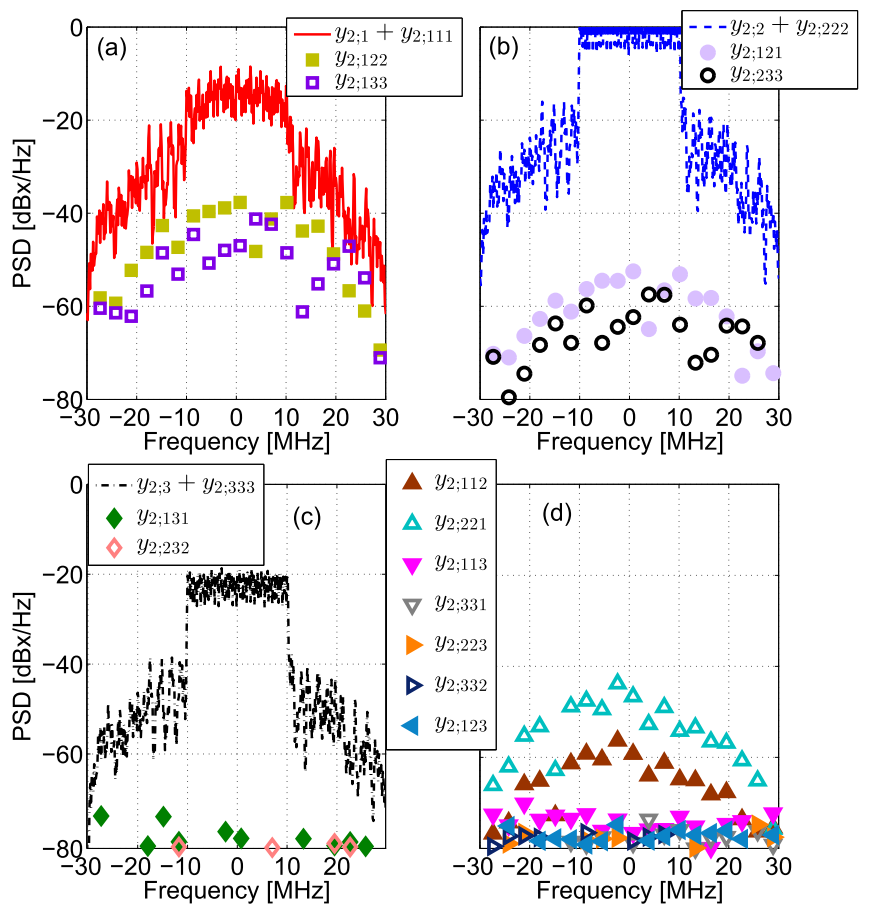

Fig. 14. PSD of the third-order kernel outputs at channel 2 for partial-input full-output crosstalk [cf., Fig. 2(e)] that partially overlap (a) $y_{2 ; 111}$, (b) $y_{2 ; 222}$, (c) $y_{2 ; 333}$, and (d) none.
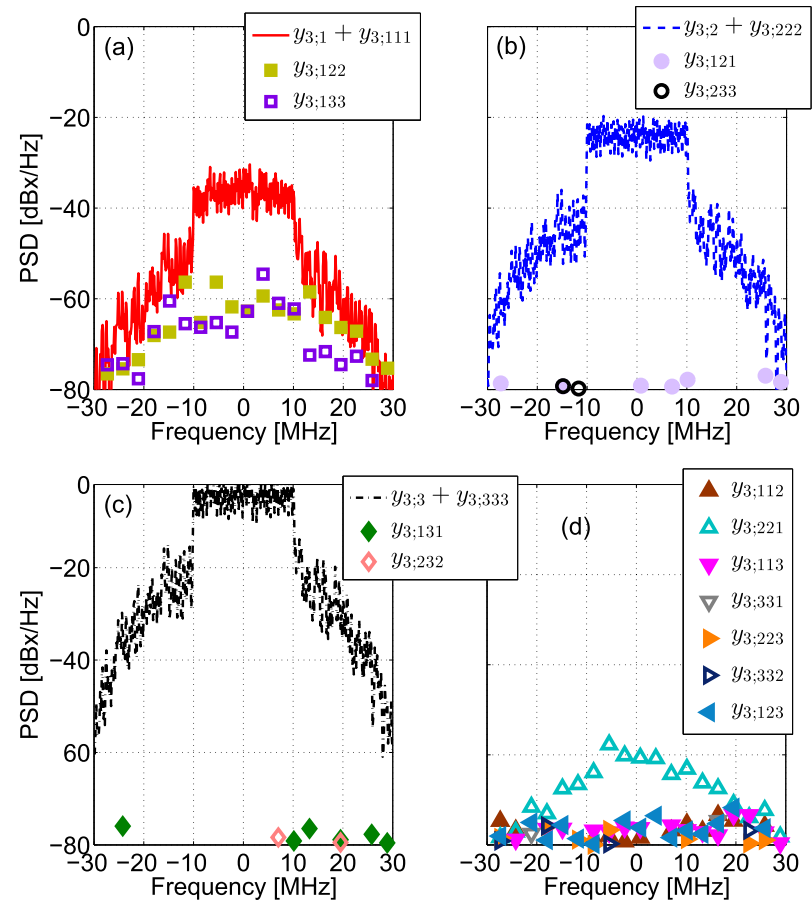

Fig. 15. PSD of the third-order kernel outputs at channel 3 for partial input full-output crosstalk [cf., Fig. 2(e)] that partially overlap (a) $y_{3 ; 111}$, (b) $y_{3 ; 222}$, (c) $y_{3 ; 333}$, and (d) none.

3) Partial-Input Partial-Output Crosstalk: Fig. 16 plots the PSD of the third-order kernel outputs at channel 1 for partial-input partial-output crosstalk. It can be noted that all the kernel outputs arise only from channel 2 since there is 

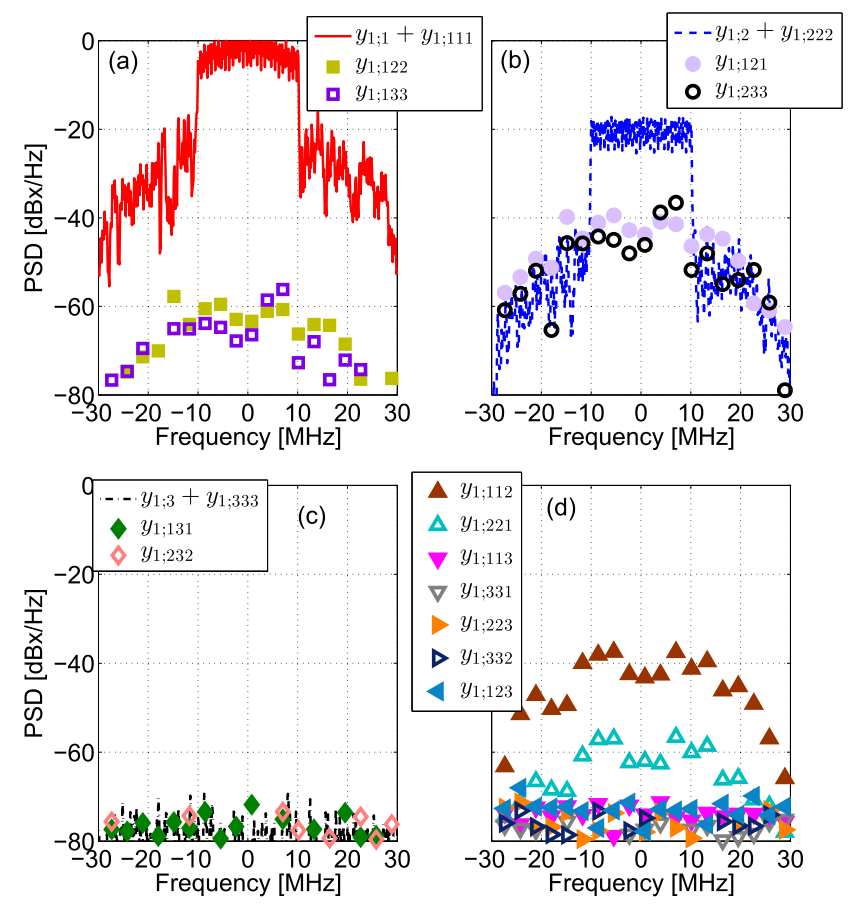

Fig. 16. PSD of the third-order kernel outputs at channel 1 for partial-input partial-output crosstalk [cf., Fig. 2(f)] that partially overlap (a) $y_{1 ; 111}$, (b) $y_{1 ; 222}$, (c) $y_{1 ; 333}$, and (d) none.
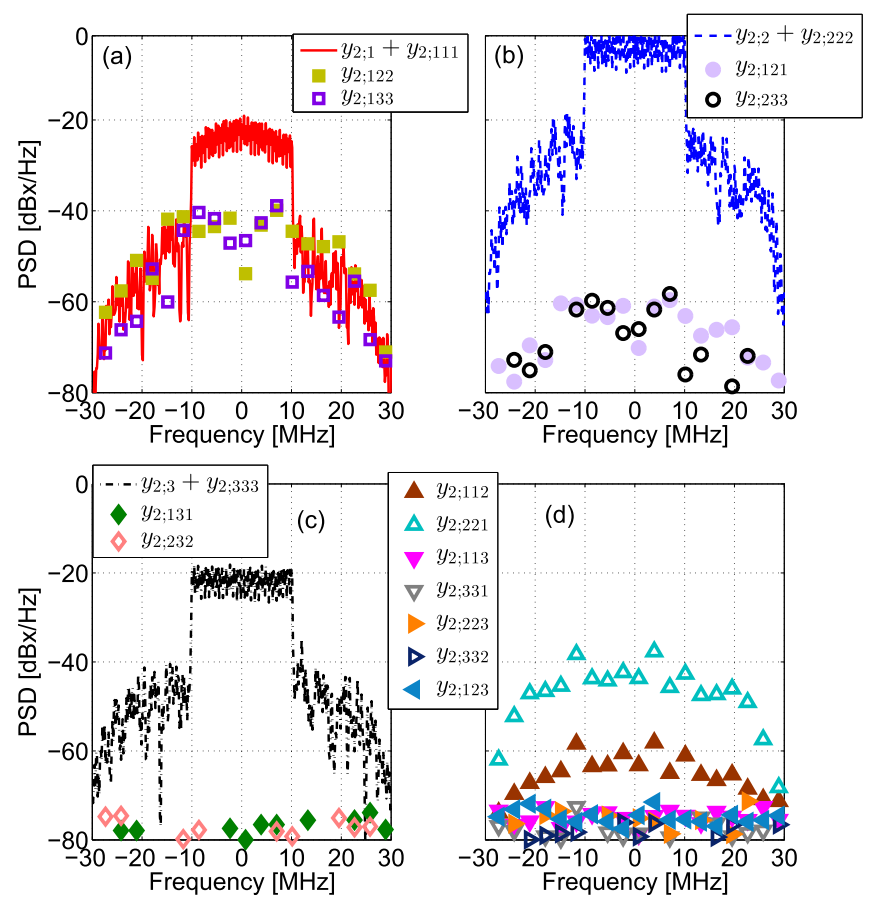

Fig. 17. PSD of the third-order kernel outputs at channel 2 for partial-input partial-output crosstalk [cf., Fig. 2(f)] that partially overlap (a) $y_{2 ; 111}$, (b) $y_{2 ; 222}$, (c) $y_{2 ; 333}$, and (d) none.

no crosstalk between channels 1 and 3, and follow the same trends as noted for full-input full-output crosstalk (cf., Fig. 8).

For channel 2, Fig. 17 shows that the self-kernels arise only from channel 3 since there is no output crosstalk from channel 1 , and crosskernels arise only from channel 1 since there is
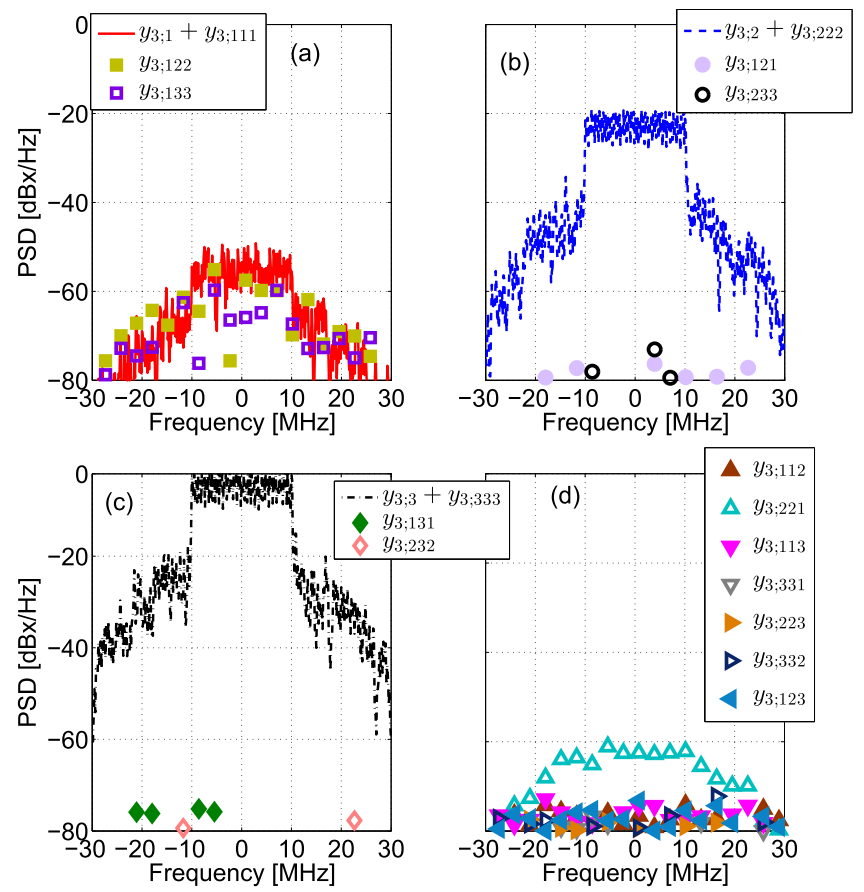

Fig. 18. PSD of the third-order kernel outputs at channel 3 for partial-input partial-output crosstalk [cf., Fig. 2(f)] that partially overlap (a) $y_{3 ; 111}$, (b) $y_{3 ; 222}$, (c) $y_{3 ; 333}$, and (d) none.

no input crosstalk on channel 3. Again, the contributions of these kernel outputs follow the same trends as noted for full input full output crosstalk (cf., Fig. 9).

Finally, for channel 3, Fig. 18 shows that no kernel outputs arise directly from channel 1 since there is no crosstalk between channels 1 and 3. Also, there are no crosskernel outputs arising directly from channel 2 since there is no input crosstalk between channels 2 and 3. Instead, only the crosskernel outputs $y_{3 ; 122}$ and $y_{3: 221}$ arise, with a weak contribution close to the noise floor. These results are in accordance with the analysis presented in Section IV-F.

\section{REFERENCES}

[1] V. Volterra, Theory of Functionals and of Integral and IntegroDifferential Equations. Mineola, NY, USA: Dover, 1959.

[2] N. Wiener, Nonlinear Problems in Random Theory. New York, NY, USA: MIT Press, 1959.

[3] M. Schetzen, The Volterra and Wiener Theories of Nonlinear Systems. New York, NY, USA: Wiley, 1980.

[4] S. Boyd and L. Chua, "Fading memory and the problem of approximating nonlinear operators with Volterra series," IEEE Trans. Circuits Syst., vol. 32, no. 11, pp. 1150-1161, Nov. 1985.

[5] A. K. Swain and S. A. Billings, "Generalized frequency response function matrix for MIMO non-linear systems," Int. J. Control., vol. 74, no. 8, pp. 829-844, 2001.

[6] L. M. Li and S. A. Billings, "Generalized frequency response functions and output response synthesis for MIMO non-linear systems," Int. J. Control, vol. 79, no. 1, pp. 53-62, 2006.

[7] Z. K. Peng, Z. Q. Lang, and S. A. Billings, "Non-linear output frequency response functions for multi-input non-linear Volterra systems," Int. J. Control, vol. 80, no. 6, pp. 843-855, 2007.

[8] W. J. Rugh, Nonlinear System Theory: The Volterra/Wiener Approach. Baltimore, MD, USA: The Johns Hopkins Univ. Press, 1981.

[9] S. Boyd, Y. Tang, and L. Chua, "Measuring Volterra kernels," IEEE Trans. Circuits Syst., vol. 30, no. 8, pp. 571-577, Aug. 1983. 
[10] L. N. O. Chua and Y. Liao, "Measuring Volterra kernels (II)," Int. J. Circuit Theory Appl., vol. 17, no. 2, pp. 151-190, 1989.

[11] C. Evans, D. Rees, L. Jones, and M. Weiss, "Periodic signals for measuring nonlinear Volterra kernels," IEEE Trans. Instrum. Meas., vol. 45, no. 2, pp. 362-371, Apr. 1996.

[12] C. Evans and D. Rees, "Nonlinear distortions and multisine signals. I. Measuring the best linear approximation," IEEE Trans. Instrum. Meas., vol. 49, no. 3, pp. 602-609, Jun. 2000.

[13] K. Vanhoenacker, T. Dobrowiecki, and J. Schoukens, "Design of multisine excitations to characterize the nonlinear distortions during FRF-measurements," IEEE Trans. Instrum. Meas., vol. 50, no. 5, pp. 1097-1102, Oct. 2001.

[14] J. C. Pedro and N. B. Carvalho, "Designing multisine excitations for nonlinear model testing," IEEE Trans. Microw. Theory Techn., vol. 53, no. 1, pp. 45-54, Jan. 2005.

[15] Z. Q. Lang, S. A. Billings, R. Yue, and J. Li, "Output frequency response function of nonlinear Volterra systems," Automatica, vol. 43, no. 5, pp. 805-816, 2007.

[16] T. R. Harper and W. J. Rugh, "Structural features of factorable Volterra systems," IEEE Trans. Autom. Control, vol. 21, no. 6, pp. 822-832, Dec. 1976.

[17] J. H. Haywood and Y. L. Chow, "Intermodulation distortion analysis using a frequency-domain harmonic balance technique," IEEE Trans. Microw. Theory Techn., vol. 36, no. 8, pp. 1251-1257, Aug. 1988.

[18] J. C. Pedro and N. B. D. Carvalho, "On the use of multitone techniques for assessing RF components' intermodulation distortion," IEEE Trans. Microw. Theory Techn., vol. 47, no. 12, pp. 2393-2402, Dec. 1999.

[19] D. H. Wisell, B. Rudlund, and D. Rönnow, "Characterization of memory effects in power amplifiers using digital two-tone measurements," IEEE Trans. Instrum. Meas., vol. 56, no. 6, pp. 2757-2766, Dec. 2007.

[20] D. Rönnow, D. Wisell, and M. Isaksson, "Three-tone characterization of nonlinear memory effects in radio-frequency power amplifiers," IEEE Trans. Instrum. Meas., vol. 56, no. 6, pp. 2646-2657, Dec. 2007.

[21] N. Björsell, P. Suchanek, P. Händel, and D. Rönnow, "Measuring Volterra kernels of analog-to-digital converters using a stepped threetone scan," IEEE Trans. Instrum. Meas., vol. 57, no. 4, pp. 666-671, Apr. 2008

[22] M. Alizadeh, S. Amin, and D. Rönnow, "Measurement and analysis of frequency-domain Volterra kernels of nonlinear dynamic $3 \times 3$ MIMO systems," IEEE Trans. Instrum. Meas., vol. 66, no. 7, pp. 1893-1905, Jul. 2017.

[23] J. J. Bussgang, L. Ehrman, and J. W. Graham, "Analysis of nonlinear systems with multiple inputs," Proc. IEEE, vol. 62, no. 8, pp. 1088-1119, Aug. 1974.

[24] Z. A. Khan, E. Zenteno, P. Händel, and M. Isaksson, "Multitone design for third order MIMO Volterra kernels," in IEEE MTT-S Int. Microw. Symp. Dig., Jun. 2017, pp. 1553-1556.

[25] S. Amin, P. Landin, P. Händel, and D. Rönnow, "Behavioral modeling and linearization of crosstalk and memory effects in RF MIMO transmitters," IEEE Trans. Microw. Theory Techn., vol. 62, no. 4, pp. 810-823, Apr. 2014

[26] S. A. Bassam, M. Helaoui, and F. M. Ghannouchi, "Crossover digital predistorter for the compensation of crosstalk and nonlinearity in MIMO transmitters," IEEE Trans. Microw. Theory Techn., vol. 57, no. 5, pp. 1119-1128, May 2009.

[27] P. M. Suryasarman and A. Springer, "A comparative analysis of adaptive digital predistortion algorithms for multiple antenna transmitters," IEEE Trans. Circuits Syst. I, Reg. Papers, vol. 62, no. 5, pp. 1412-1420, May 2015.

[28] E. Zenteno, R. Piazza, B. S. M. R. Rao, D. Rönnow, and B. Ottersten, "Low complexity predistortion and equalization in nonlinear multicarrier satellite communications," EURASIP J. Adv. Signal Process, vol. 2015, no. 1, pp. 1-15, 2015.

[29] Z. A. Khan, E. Zenteno, P. Händel, and M. Isaksson, "Digital predistortion for joint mitigation of $\mathrm{I} / \mathrm{Q}$ imbalance and MIMO power amplifier distortion," IEEE Trans. Microw. Theory Techn., vol. 65, no. 1, pp. 322-333, Jan. 2017.

[30] Y. Palaskas et al., "A 5-GHz 108-Mb/s $2 \times 2$ MIMO transceiver RFIC with fully integrated $20.5-\mathrm{dBmP}_{1 d B}$ power amplifiers in 90-nm CMOS," IEEE J. Solid-State Circuits, vol. 41, no. 12, pp. 2746-2756, Dec. 2006.

[31] W.-C. Hua et al., "Coupling effects of dual SiGe power amplifiers for 802.11n MIMO applications," in Proc. IEEE Radio Freq. Integr. Circuits (RFIC) Symp., Jun. 2006, pp. 4-7.
[32] F. F. Dai, Y. Shi, J. Yan, and X. Hu, "MIMO RFIC transceiver designs for WLAN applications," in Proc. 7th Int. ASIC Conf., Oct. 2007, pp. $348-351$.

[33] T. Sadeghpour, R. A. Alhameed, N. T. Ali, I. T. E. Elfergani, Y. Dama, and O. O. Anoh, "Linear and nonlinear crosstalk in MIMO OFDM transceivers," in Proc. 18th IEEE Int. Conf. Electron., Circuits, Syst. (ICECS), Dec. 2011, pp. 504-507.

[34] D. M. Pozar, Microwave Engineering. New York, NY, USA: Wiley, 1998.

[35] D. Hummels and R. Gitchell, "Equivalent low-pass representations for bandpass Volterra systems," IEEE Trans. Commun., vol. 28, no. 1, pp. 140-142, Jan. 1980.

[36] Holzworth Instrumentation, "Ultra low phase noise-Phase coherent synthesizers," Microw. J., vol. 10, pp. 48-50, Aug. 2009.

[37] L. Anttila, M. Valkama, and M. Renfors, "Frequency-selective I/Q mismatch calibration of wideband direct-conversion transmitters," IEEE Trans. Circuits Syst. II, Exp. Briefs, vol. 55, no. 4, pp. 359-363, Apr. 2008.

[38] Z. Zhu, X. Huang, and H. Leung, "Joint I/Q mismatch and distortion compensation in direct conversion transmitters," IEEE Trans. Wireless Commun., vol. 12, no. 6, pp. 2941-2951, Jun. 2013.

[39] E. Zenteno, S. Amin, M. Isaksson, P. Händel, and D. Rönnow, "Combating the dimensionality of nonlinear MIMO amplifier predistortion by basis pursuit," in Proc. 44th Eur. Microw. Conf. (EuMC), Oct. 2014, pp. 833-836.

[40] I. Jaffel, O. Taouali, M. F. Harkat, and H. Messaoud, "Kernel principal component analysis with reduced complexity for nonlinear dynamic process monitoring," Int. J. Adv. Manuf. Technol., vol. 88, no. 9, pp. 3265-3279, Feb. 2017.

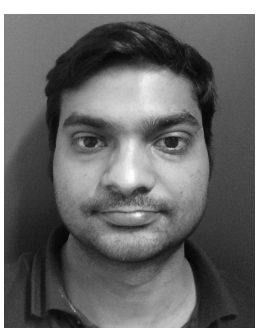

Zain Ahmed Khan (S'13) received the B.S. degree in electronics engineering from the Ghulam Ishaq Khan Institute, Swabi, Pakistan, in 2005, and the M.S. degree in communication and signal processing from Technical University Ilmenau, Illmenau, Germany, in 2013. He is currently pursuing the $\mathrm{Ph} . \mathrm{D}$. degree at the Department of Information Science and Engineering, KTH Royal Institute of Technology, Stockholm, Sweden.

$\mathrm{He}$ is currently with the Department of Electronics, Mathematics, and Natural Sciences, University of Gävle, Gävle, Sweden. He is involved in the characterization, behavioral modeling, and digital predistortion of nonlinear RF MIMO and massive MIMO transmitters impaired by crosstalk.

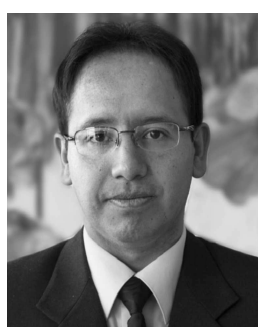

Efrain Zenteno (S'10-M'16) received the B.S. degree in electronics engineering from the Universidad San Agustn, Iloilo City, Philippines, in 2005, the M.Sc. degree in electronics and telecommunications from the University of Gävle, Gävle, Sweden, in 2008, and the Ph.D. degree from the KTH Royal Institute of Technology, Stockholm, Sweden, in 2015 .

$\mathrm{He}$ is currently with Universidad Catolica San Pablo, Arequipa, Peru, where he is responsible for teaching and research activities with the Electronics and Telecommunications Department. His current research interests include measurements and signal processing for communications. 


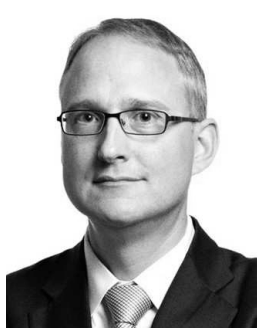

Peter Händel (S'88-M'94-SM'98) received the Ph.D. degree from Uppsala University, Uppsala, Sweden, in 1993.

From 1987 to 1993, he was with Uppsala University. From 1993 to 1997, he was with Ericsson AB, Kista, Sweden. From 1996 to 1997, he was a Visiting Scholar with the Tampere University of Technology, Tampere, Finland. Since 1997, he has been with the KTH Royal Institute of Technology, Stockholm, Sweden, where he is currently a Professor of signal processing with the Department of Information Science and Engineering. From 2000 to 2006, he held an adjunct position with the Swedish Defence Research Agency. He has been a Guest Professor with the Indian Institute of Science, Bangalore, India, and the University of Gävle, Gävle, Sweden.

Dr. Händel was a recipient of the Best Survey Paper Award of the IEEE Intelligent Transportation Systems Society in 2013. He has served as an Associate Editor for the IEEE TRANSACTIONS ON Signal PROCESSING.

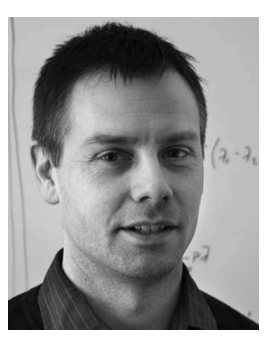

Magnus Isaksson (S'98-M'07-SM'12) received the M.Sc. degree in microwave engineering from the University of Gävle, Gävle, Sweden, in 2000, the Licentiate degree from Uppsala University, Uppsala, Sweden, in 2006, and the Ph.D. degree from the KTH Royal Institute of Technology, Stockholm, Sweden, in 2007.

In 2012, he was appointed as a Docent in telecommunications with the KTH Royal Institute of Technology, Stockholm, Sweden. From 1989 to 1999 ,

he was with the Televerket, Stockholm, where he was involved in communication products. Since 1999, he has been with the Department of Electronics, Mathematics, and Natural Sciences, University of Gävle, where he is currently a Professor and the Head of the Electronics Department. $\mathrm{He}$ is currently the Head of research within the fields of mathematics, and natural sciences with the University of Gävle. He has authored or co-authored many peer-review journal papers, books, and conference proceedings in the area. His current research interests include signal processing algorithms for radio frequency measurements and characterization, modeling, and compensation of nonlinear microwave devices and systems. 